\title{
A robotic model to investigate human motor control
}

\author{
Tommaso Lenzi • Nicola Vitiello • Joseph McIntyre • \\ Stefano Roccella · Maria Chiara Carrozza
}

Received: 29 March 2010 / Accepted: 15 June 2011 / Published online: 19 July 2011

(C) The Author(s) 2011. This article is published with open access at Springerlink.com

\begin{abstract}
The role of the mechanical properties of the neuromuscular system in motor control has been investigated for a long time in both human and animal subjects, mainly through the application of mechanical perturbations to the limb during natural movements and the observation of its corrective responses. These methods have provided a wealth of insight into how the central nervous system controls the limb. They suffer, however, from the fact that it is almost impossible to separate the active and passive components of the measured arm stiffness and that the measurement may themselves alter the stiffness characteristic of the arm. As a complement to these analyses, the implementation of a given neuroscientific hypothesis on a real mechanical system could overcome these measurement artifact and provide a tool that is, under full control of the experimenter, able to replicate the relevant functional features of the human arm. In this article, we introduce the NEURARM platform, a robotic arm intended to test hypotheses on the human motor control system. As such, NEURARM satisfies two key requirements. First, its kinematic parameters and inertia are similar to that of the human arm. Second, NEURARM mimics the main physical features of the human actuation system, specifically, the use of tendons to transfer force, the presence of antagonistic muscle pairs, the passive elasticity of muscles in the absence of any neural feedback and the non-linear elastic behaviour. This article presents the design and characterization of the NEURARM actuation system. The resulting mechanical behaviour, which has been tested in joint and
\end{abstract}

T. Lenzi $(\bowtie) \cdot$ N. Vitiello $\cdot$ S. Roccella $\cdot$ M. C. Carrozza

The BioRobotics Institute, Scuola Superiore Sant'Anna, Pisa, Italy

e-mail: lenzi@ieee.org

J. McIntyve

Centre d'Etudes de la Sensorimotricité (CESEM), Université Paris

Descartes-CNRS, Paris, France
Cartesian space under static and dynamic conditions, proves that the NEURARM platform can be exploited as a robotic model of the human arm, and could thus represent a powerful tool for neuroscience investigations.

Keywords Neurorobotics - Anthropomorphic robotic arm $\cdot$ Equilibrium point hypothesis $\cdot$ Impedance control

\section{Introduction}

A pair of muscles powering the human joint in an antagonistic configuration exemplifies the main difference between standard industrial robots and biological motor systems. Since muscles have a natural stiffness that varies with the muscle activation level, the central nervous system (CNS) can generate stable equilibrium postures, towards which the arm is attracted, by properly regulating the activation levels of antagonistic muscles (Hogan 1984) The elastic properties of muscles contribute to the finite stiffness/compliance properties of the limb and to the stability of the neuro-musculo-skeletal system in the face of significant feedback delays and even allows for the generation of target movements in the absence of sensory feedback, by shifting the equilibrium point (Polit and Bizzi 1979). Moreover, the ability to modulate the stiffness of the limb is fundamental to the control of stable interactions with the environment (Colgate and Hogan 1988) leading to the theory of 'impedance control' (Hogan 1985a,b,c).

The intrinsic spring-like muscles of the arm in theory allow the human motor system to generate stable postures and movements even in the absence of feedback control. Considering a single joint, the basic idea is that, working against each other, the two opposing spring-like muscles can establish a joint equilibrium position (EP). When the joint is at the EP, the net force and torque acting on it is zero. If moved to the EP and released, the joint will stay there. If displaced 
away from the EP by an external force and then released, the spring-like muscle properties will pull the limb back towards the EP. The EP is therefore a stable attractor. Control theories based on the presence of the EP in biological motor systems (Hogan 1984; Hogan et al. 1987; Bizzi et al. 1984; Feldman $1986,1966)$ suggest that movements are programmed as a shift of EPs rather than through an explicit computation of forces. Thus, there is no need to solve the 'inverse dynamics problem' for calculating the torque required to move the arm on the desired trajectory.

The early EP hypotheses are, however, admittedly too simple to explain the full complexity of human dynamic behaviours, for which more sophisticated model-based control schemes are almost certainly engaged as well. Nevertheless, early studies of equilibrium-point control hypotheses provided critical insights underlining the importance of the intrinsic properties of the muscles and of impedance in the stable control of the arm. EP, impedance and model-based control mechanisms certainly work together in the overall control of the arm. Experiments addressing questions about limb impedance have been tested extensively in human and animal subjects, primarily by applying mechanical perturbations (impulses, vibrations, etc.) to the limb during natural movements and observing the corrective responses of the limb (Burdet et al. 2001). These methods have provided a wealth of insight into how the CNS controls the mechanical behaviour of the limb. They suffer, however, from the fact that the applied perturbations may themselves change the stiffness characteristics of the arm, a highly non-linear system, which could lead to corrupted observations of the limb behaviour.

A possible way to overcome the measurement artifact problem consists of simulating the human arm movements using mathematical models describing the behaviour of the muscle-skeletal system. The simulation results can then be compared with the human performance, thus obtaining important information about the internal mechanism responsible for the human arm behaviour (Flash 1987). This research methodology represents a powerful tool for neuroscience investigation. However, mathematical models of complex physical phenomena, such as the interaction between the human arm and the external environment, always present a discrepancy with the real world. This discrepancy could either be due to un-modelled dynamics of the interested phenomena or to the impossibility of finding the exact parameters of the model that reproduce the characteristics of the real-world phenomena. Discrepancies such as these could result in a divergence between the simulation result and real-world behaviour, reducing the model usefulness.

As a complement to mathematical analyses, the implementation of a given neuroscientific hypothesis on a real mechanical system can avoid the need of modelling parts of the phenomena of interest. For example, in the case of physical interaction between the human arm and the external environment, both the external environment properties and the interaction dynamics need not be modelled, thus reducing the risk of misrepresentation due to inadequate models of the real physical world.

The aim of this study, therefore, is to produce a robotic model of the human arm that replicates the key mechanical behaviour of the human neuromuscular system. As such, an accurate robotic model of the human arm could provide a tool under the full control of the experimenter, reproducing the main functional features of the human arm and being able to interact with the same physical environment as the human.

In order to develop an appropriate robotic model of the human arm, two key requirements should be satisfied:

1. the robot kinematic parameters and inertia should be similar to that of the human being;

2. the robot actuation should mimic the main physical features of the human actuator system, such as:

2.1 the use of tendons to transfer force;

2.2 passive elasticity of muscles in the absence of any neural feedback;

2.3 implementation of antagonistic pairs of muscle;

2.4 non-linearity of the elastic behaviour allowing modulation of net stiffness through co-activation of opposing muscles.

These criteria relate to the constituent components of the human described at the level of the joints and mechanical linkages. If these criteria are met, the more global features of the dynamical system, such as the endpoint impedance and the movements induced by a motor command, should emerge. If, indeed, this goal is reached, one can use the thusdesigned robotics system to test hypotheses about human motor control, allowing one to distinguish between the dynamical properties of the skeletal elements and actuators that contribute to the control of the limb from the active neural processes that the CNS uses to produce movements. This reasoning has driven the research activities that we describe here. The NEURARM bio-mimetic platform has been designed and implemented by reproducing key features of the human arm as identified at the level of joints and muscles. We then used the NEURARM platform to replicate and compare results with two well-known studies (one primarily experimental, one primarily based on numerical modelling) that have previously been performed to identify the impedance characteristics of the human upper arm. In doing so, we confirm the early results from the human experiments. More importantly, we use this comparison to validate the NEURARM as a useful platform that can be used to test more recent theories about human motor control. 
Fig. 1 The NEURARM platform 2-link, 2-dof planar manipulator

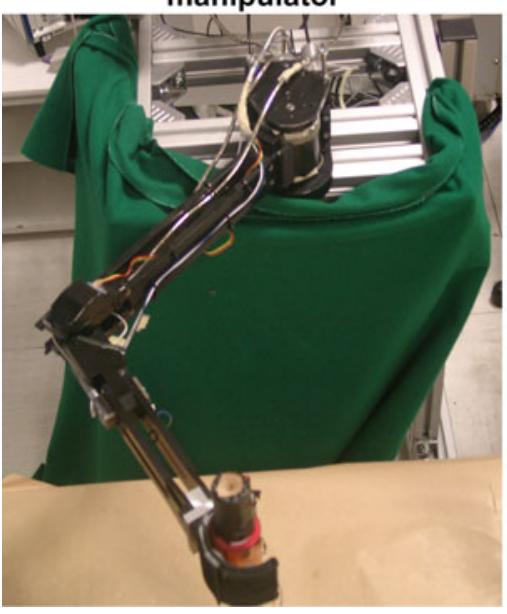

Remote actuation block

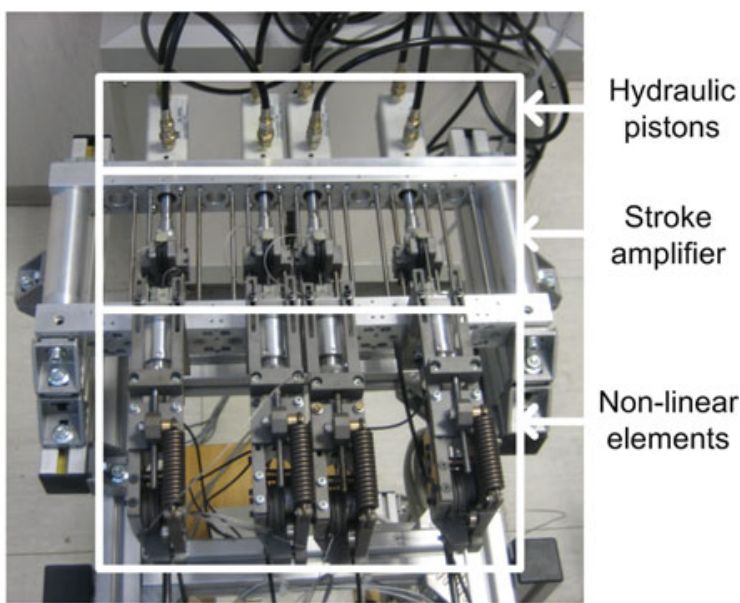

\section{Design of the NEURARM}

State-of-the-art industrial anthropomorphic robot arms currently fail to replicate the muscle spring-like properties that characterize biological systems because of the use of rigid, non-backdrivable transmission systems in the mechatronic design. Recent advances in this field present a bio-inspired actuation system based on antagonistic compliant actuators. For instance, Filippini et al. (2008) developed the variable stiffness actuators (VSA) to reduce the risk of injury in case of impact with the human being. Migliore et al. (2005) implemented an equilibrium-point-hypothesis-based openloop controller for a single joint tendon-driven robotic system. Koganezawa et al. (2006) developed the actuator with non-linear elastic system (ANLES) to exploit the energy efficiency and the intrinsic stability of the antagonist compliant actuators. Nevertheless, these robotic manipulators still do not accurately model the elastic behaviour of the human arm. Indeed, the force and stiffness ranges achieved by these actuators differ from that of the human arm, the links length mass and inertia are not consistent with that of human being, and their application is limited to a single degree-of-freedom. A different approach for developing bio-inspired actuation system consists of the use of actuators that replicate some intrinsic muscle properties, such as the pneumatic artificial muscle (PAM) and shape memory alloys (SMA) (Caldwell et al. 1995; Tondu et al. 2005; Selden et al. 2006). However, these devices have intrinsic disadvantages such as difficult controllability and low bandwidth, which limit their usability whilst performing fast movements.

In Cattin et al. (2008), Lenzi et al. (2009) and Vitiello et al. (2010), we presented the two-link, two degree-of-freedom planar anthropomorphic robotic arm NEURARM, designed for the purpose of making a realistic model of the human arm for neuroscience investigations. This planar system (see
Fig. 1) is a gross simplification of the complexity of the human arm: its dofs are reduced to a minimum both in terms of kinematics and actuation (i.e., two dofs and four actuators). The system is complex enough, however, to address essential questions about human behaviour. Specifically, the two-joint linkage provides significant non-linear kinematics, statics and dynamics and the tendon-driven antagonist actuation system supplies redundancy in terms of both force and torque production.

Previous incarnations of the NEURARM already replicated some of the main functional features of the human upper limb (i.e., tendon-driven agonist-antagonist actuation, mass, inertia, dynamic performance, sensory system for detecting tendon forces, joint angles and actuators elongation) but did not successfully reproduce the muscle's spring-like properties. To overcome these limitations, the transmission and control system of the NEURARM were updated as follows:

1. A new agonist-antagonist tendon-driven joint that includes a novel non-linear elastic element that mimics the passive muscle elasticity was conceived and implemented as part of the NEURARM transmission system.

2. A joint-level position and stiffness control was developed and tested to investigate hypotheses on 'equilibrium point' and 'impedance' control.

Reports on these developments, and tests of their efficacy, are reported here.

\subsection{Design goals: variable-stiffness, antagonistically actuated joints}

The length-tension characteristic of a muscle, or of a combined muscle-reflex, is that of a nonlinear spring. The important property of these springs is that the stiffness of the 
(a)

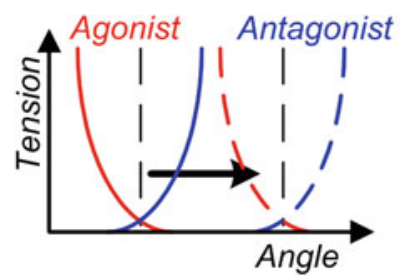

(b)

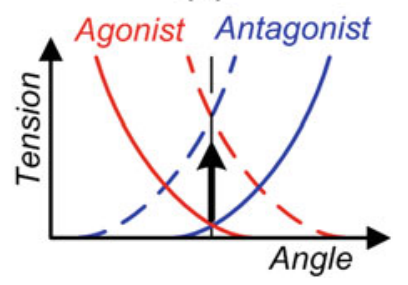

Fig. 2 Two non-linear springs acting on the joint in antagonist configuration permit to control both the joint position and the joint stiffness by regulating the rest lengths of the opposing non-linear elements

length/tension curve increases as the tension increases, at least over a certain usable range. This property allows one to control both the net stiffness and the equilibrium point for a pair of opposing muscles, even if the control signal serves only to shift the rest-length of each nonlinear spring. Figure 2 shows how two idealized muscles, behaving as two non-linear springs, generate a convergent torque field on the powered joint. By properly changing the two rest lengths, it is possible to change the equilibrium point (i.e., the joint equilibrium posture), as shown in Fig. 2a, and/or the slope of the torque field (i.e., the joint stiffness), as shown in Fig. 2b. ${ }^{1}$

The length-tension characteristics that we set out to reproduce were inspired by the so-called invariant characteristic that humans show around joints of the upper limb. Feldman (1966) measured these characteristics by asking subjects to actively exert a defined level of force against a mechanical stop. The mechanical stop was then gradually moved so as to allow the muscle to shorten and release the tension, whilst subjects were instructed to not consciously intervene. The length-tension curves recorded in these experiments, which are presumed to result from a combination of muscle elastic properties and stretch-reflex activity, showed the desirable characteristic that the muscle stiffness decreased as the tension in the muscle decreased. It is this feature that we set out to mimic in the design of the NEURARM actuator system. Although this characterisation of length-tension properties represents a gross simplification of the complex behaviour exhibited by human muscles, we argue that the design specified here captures the essential characteristics of the biological systems that are necessary to test hypotheses about at least a certain class of human arm movements (see Sect. 5).

\footnotetext{
1 The equilibrium point hypothesis has been proposed at least in two main formulations: the alpha model, proposed by Bizzi and colleagues and Feldman's lambda model. These two versions suggest a different mechanism underling the EP generation and maintenance, but they are both in accordance with the idea that the viscoelastic properties of the neuromuscular system play a fundamental role in the control of the human arm.
}

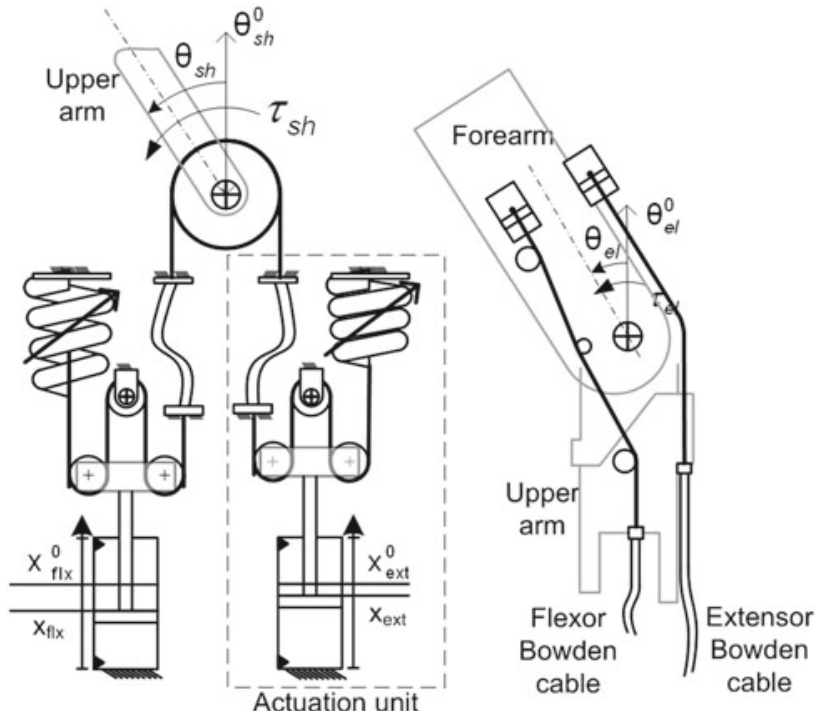

(a)

(b)

Fig. 3 (a) NEURARM joint antagonistic actuation scheme: each actuation unit is composed of three elements: the hydraulic piston-stroke amplifier, the non-linear elastic element and the steel cable which, by means of Bowden cable, transmits the power to the joint; (b) NEURARM elbow joint design: the two antagonistic cables transmit the torque to the joint by acting directly on the forearm link through cable clamps; whilst the extensor cable has a constant moment arm, the flexor has a non-constant and non-linear moment arm; this feature, which replicates the human biceps attachment, is obtained by routing the cable through the pins $\mathrm{A}, \mathrm{B}$ and $\mathrm{C}$

\subsection{Design overview}

The NEURARM joint actuation system, preliminarily presented in Vitiello et al. (2008) for the shoulder joint, and here extended to the elbow, replicates the human musculoskeletal system configuration by means of two antagonistic compliant actuators. As illustrated in Fig. 3a, each agonist/antagonist unit consists of three functional elements:

1. A non-linear elastic element emulating the muscle's passive elastic behaviour.

2. A linear hydraulic actuator combined with a stroke amplifier to mimic the contractile capability of the muscle. These two elements allow the regulation of the rest length of the non-linear elastic element. The hydraulic piston is the active component of the transmission system, whilst the stroke amplifier is used to transform a piston displacement $\Delta x$ into a cable displacement $\Delta l=a_{\mathrm{f}} \cdot \Delta x=4 \cdot \Delta x$ (where $a_{\mathrm{f}}$ is the amplification factor). This latter element satisfies the system requirement in terms of contraction velocity and force generation, and allows one to achieve performance similar to that of the human arm.

3. A steel cable transmitting the force to the NEURARM joint by means of a Bowden cable. Regarding the 
shoulder joint, the two antagonist steel cables act on a pulley, so that the moment arms are equal and constant for each joint angle (see Fig. 3a). On the contrary, for the elbow joint (Fig. 3b), the two cables attach directly on the forearm. The elbow flexor cable follows a particular geometry, generating a variable moment arm with respect to the joint position. This non-linear function emulates that of the human elbow joint and represents a further element of bio-inspiration on our platform.

Both elements 1 and 2 are located remotely from the robot. This solution satisfies requirements for the mass and inertia of the links without affecting the system's capability to functionally emulate the human musculoskeletal system. This would not have been possible with an on-board actuation solution. Moreover, thanks to the low weight and high flexibility of the Bowden cables, the power is transferred to the joints without affecting the arm kinetics and kinematics. In the following, we describe the individual elements of the NEURARM design and explain how these elements come together to allow for feedforward impedance control at the level of the joints.

\subsection{Design of the NEURARM non-linear springs}

The design of the non-linear elastic element was of crucial importance to mimic the human muscle characteristics. Its main design requirements were:

1. the force $(F)$ versus elongation $(\Delta l)$ characteristic curve should be well approximated by a quadratic function:

$F(\Delta l)=a_{1} \Delta l^{2}+a_{2} \Delta l$

such that the force increases with elongation and the stiffness $(\mathrm{d} F / \mathrm{d} L)$ increases with force.

2. the linear stiffness of the spring, coupled with the tendon transmission around the joints should result in a joint stiffness range for the shoulder and the elbow joint of about $40 \mathrm{Nm} \mathrm{rad}^{-1}$, based on measured values of human arm stiffness in a static position (Mussa-Ivaldi et al. 1985) or during movement (Gomi and Kawato 1997; Burdet et al. 2000).

Starting from a linear tension spring, we designed a lowfriction mechanism to obtain the non-linear elastic behaviour. The mechanism works in a two-stage fashion. First, it establishes a non-linear relationship between the displacement of the cable $(\Delta l)$ and the elongation of the linear tension spring $(\Delta s)$. Second, the force exerted by the linear-tension spring $F_{\Delta s}$ is transformed into a force on the cable $F_{\Delta l}$ by a cam mechanism.
As illustrated in the schematic representation of Fig. 4a, the mechanism consists of six elements, the working principles of which can be summarized in five steps:

1. the steel cable is deflected by an idler pulley (body 1) and then wrapped around a reel (body 2) which is fixed with a cam (body 3);

2. the cam transmits the force and the movement to a bar (body 5) by means of an idler wheel (body 4), minimizing the friction;

3. the bar is hinged down on the frame and is connected at its opposite extremity to the tension spring (body 6), which is hinged on the frame;

4. a displacement $\Delta l$ of the cable rotates the reel and consequently the cam;

5. the cam moves the bar via the idle wheel, and so the tension spring is stretched of $\Delta s$.

Hereafter, by referring to the schematic representation in Fig. $4 c$, the kinematic and static analysis of the mechanism is reported. The starting point for the kinematic analysis is the cable elongation $\Delta l$, which determines a rotation of the reel $\Delta \theta_{2}$ and consequently a rotation of the cam $\Delta \theta_{3}$, so that:

$\Delta \theta_{3}=\Delta \theta_{2}=-\frac{\Delta l}{R_{2}}$

where $R_{2}$ is the reel radius. Indicating with $\theta_{2}^{0}=\theta_{3}^{0}$ the angle of the reel and the cam for which $\Delta l=0$, Eq. 2 becomes:

$\theta_{3}=\theta_{3}^{0}+\Delta \theta_{3}=\theta_{3}^{0}-\frac{\Delta l}{R_{2}}$

The cam and the idle wheel interact on a single point, the intersection of the common tangent line and the segment $\mathrm{CO}_{4}$, which connects their curvature centres. The distance $\overline{\mathrm{CO}_{4}}$ is therefore the sum of the cam radius $R_{3}$ and the idle wheel radius $R_{4}$. As a consequence, the segments $\mathrm{O}_{3} \mathrm{C}, \mathrm{CO}_{4}$ and $\mathrm{O}_{3} \mathrm{O}_{5}$ behave as a four bar mechanism. The relationship between the cam rotation $\theta_{3}$ and the bar rotation $\theta_{5}$ (with respect to the horizontal axis) is:

$$
\begin{aligned}
A= & \overline{O_{3} O_{3}^{\prime}}+\overline{O_{3} C} \cdot \sin \left(\theta_{3}\right)-\overline{O_{4}^{\prime} O_{5}} \cdot \sin \left(\theta_{5}\right) \\
& -\overline{O_{4} O_{4}^{\prime}} \cdot \cos \left(\theta_{5}\right) \\
B= & \overline{O_{3}^{\prime} O_{5}}+\overline{O_{4}^{\prime} O_{5}} \cdot \cos \left(\theta_{5}\right)-\overline{O_{3} C} \cdot \cos \left(\theta_{3}\right) \\
& -\overline{O_{4} O_{4}^{\prime}} \cdot \sin \left(\theta_{5}\right) \\
\left(R_{3}+\right. & \left.R_{4}\right)=\sqrt{A^{2}+B^{2}}
\end{aligned}
$$

Equation 4 can be used to calculate the bar angular rotation $\theta_{5}$ as a function of $\theta_{3}$, and consequently, by applying Eq. 3, as a function of the cable elongation $\Delta l$.

The linear tension spring deformation $\Delta s$ depends on the bar orientation $\theta_{5}$ according to the following geometrical considerations. The linear tension spring length $s$ is equal to: 
(a)

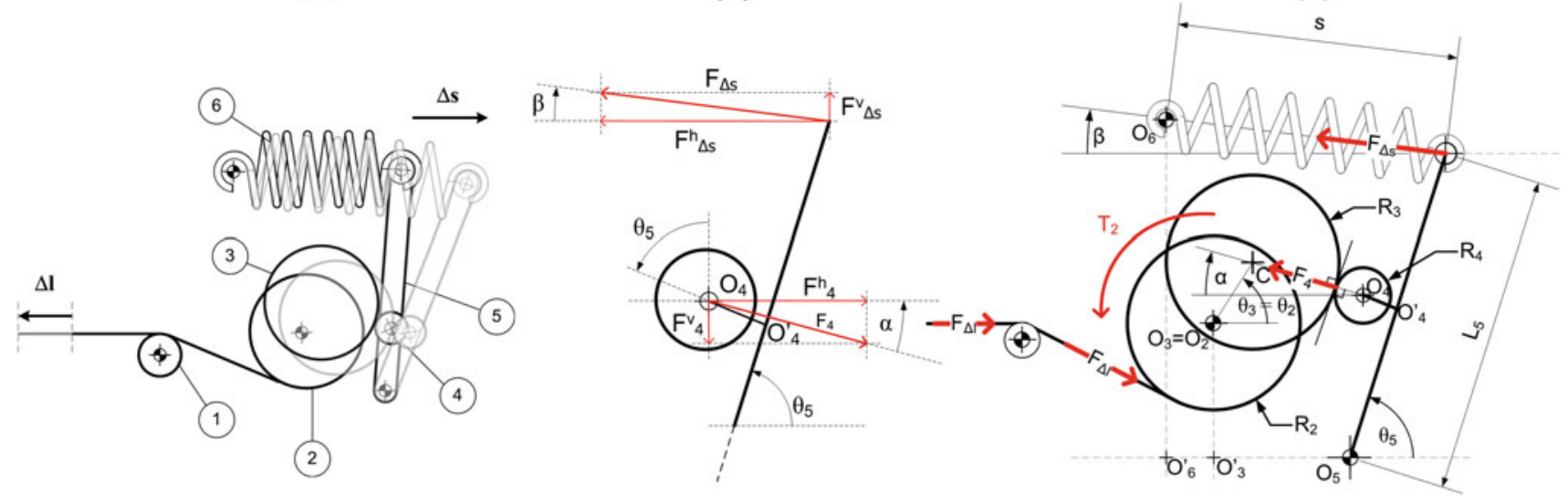

(b)

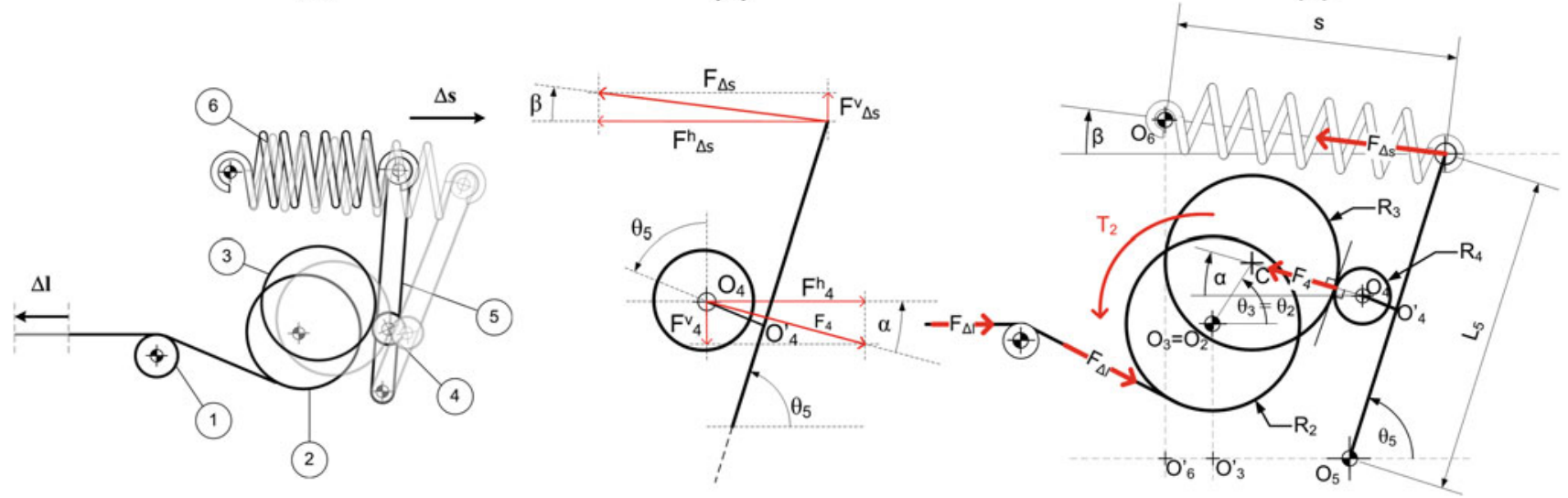

(c)

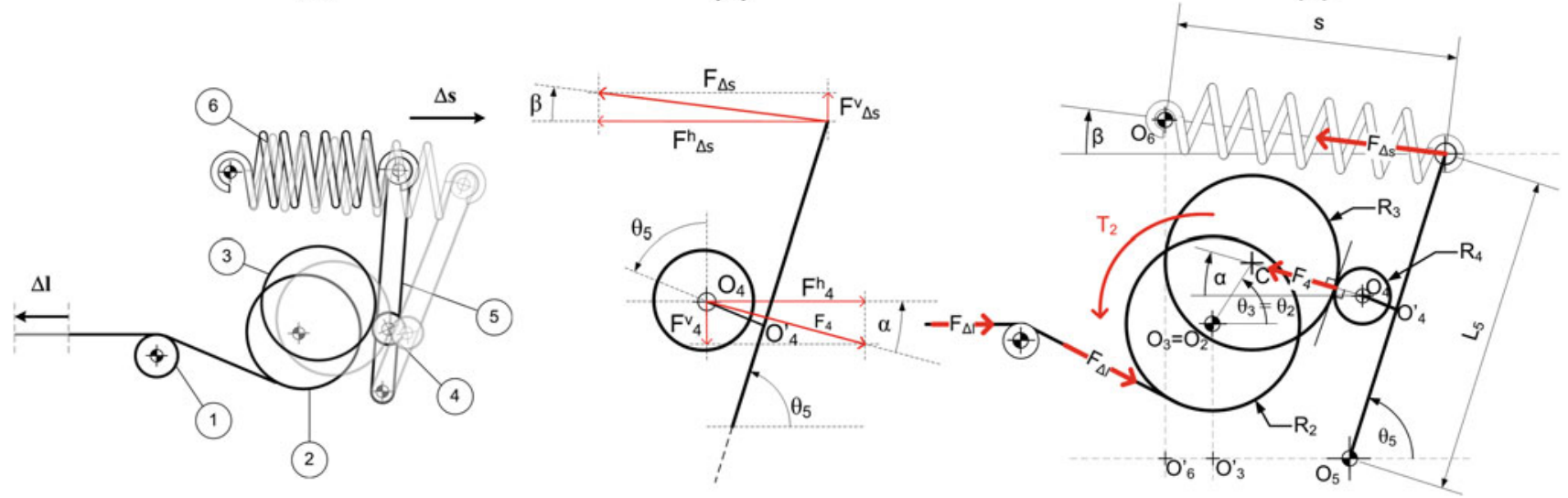

Fig. 4 Schematic representation of the non-linear elastic element: (a) all the mechanism bodies enumerated, (b) detail of the geometrical model and (c) geometrical model

$s=\sqrt{\left(\overline{O_{6}^{\prime} O_{5}}+L_{5} \cdot \cos \left(\theta_{5}\right)\right)^{2}+\left(\overline{O_{6} O_{6}^{\prime}}-L_{5} \cdot \sin \left(\theta_{5}\right)\right)^{2}}$

where $L_{5}$ is the bar length. The resulting spring deformation is then obtained by introducing $s_{0}$, which is the rest length of the tension linear spring:

$$
\begin{aligned}
\Delta s & =s-s_{0} \\
& =\sqrt{\left(\overline{O_{6}^{\prime} O_{5}}+L_{5} \cdot \cos \left(\theta_{5}\right)\right)^{2}+\left(\overline{O_{6} O_{6}^{\prime}}-L_{5} \cdot \sin \left(\theta_{5}\right)\right)^{2}}-s_{0}
\end{aligned}
$$

The elastic force provided by the linear tension spring as a consequence of a deformation $\Delta s$ is:

$F_{\Delta s}=K_{L S} \Delta s$

where $K_{L S}$ is the spring stiffness. The elastic force $F_{\Delta s}$ is transmitted by the bar/cam mechanism to the cable as a force $F_{\Delta l}$ opposing to the elongation $\Delta l$. The mathematical relation describing the force equilibrium is hereafter reported.

The spring force spans an angle $\beta$ with respect to the horizontal axis

$\beta=\arctan \left(\frac{\overline{O_{6} O_{6}^{\prime}}-L_{5} \cdot \sin \left(\theta_{5}\right)}{\overline{O_{6}^{\prime} O_{5}}+L_{5} \cdot \cos \left(\theta_{5}\right)}\right)$

whilst the interaction force between the bar (body $\mathbf{5}$ ) and the cam (body 3) trough the idle wheel (body 4) $\left(F_{4}\right)$ describes an angle $\alpha$

$\alpha=\arctan \left(\frac{\overline{O_{3} O_{3}^{\prime}}+\overline{O_{3} C} \cdot \sin \left(\theta_{3}\right)-\overline{O_{4}^{\prime} O_{5}} \cdot \sin \left(\theta_{5}\right)-\overline{O_{3}^{\prime} O_{5}}+\overline{O_{4}^{\prime} O_{5}^{\prime}} \cdot \cos \left(\theta_{5}\right)}{\cos \left(\theta_{5}\right)-\overline{O_{3} C} \cdot \cos \left(\theta_{3}\right)-\overline{O_{4} O_{4}^{\prime}} \cdot \sin \left(\theta_{5}\right)}\right)$

The $\beta$ and $\alpha$ angles can be used to solve the rotational static equilibrium equation of the body $\mathbf{5}$ with respect to the point $\mathrm{O}_{5}$ :

$$
\begin{aligned}
F_{4}^{\mathrm{h}} & \left(\overline{O_{5} O_{4}^{\prime}} \sin \left(\theta_{5}\right)+\overline{O_{4} O_{4}^{\prime}} \cos \left(\theta_{5}\right)\right) \\
& +F_{4}^{\mathrm{v}}\left(\overline{O_{5} O_{4}^{\prime}} \cos \left(\theta_{5}\right)-\overline{O_{4} O_{4}^{\prime}} \sin \left(\theta_{5}\right)\right) \\
= & F_{\Delta s}^{\mathrm{h}} L_{5} \sin \left(\theta_{5}\right)+F_{\Delta s}^{\mathrm{v}} L_{5} \cos \left(\theta_{5}\right) \\
& F_{4} \cdot\left(\overline{O_{5} O_{4}^{\prime}} \cdot \sin \left(\alpha+\theta_{5}\right)+\overline{O_{4} O_{4}^{\prime}} \cdot \cos \left(\alpha+\theta_{5}\right)\right) \\
= & F_{\Delta s} L_{5}\left(\sin \left(\beta+\theta_{5}\right)\right) \\
F_{4}= & \frac{F_{\Delta s} \cdot L_{5} \cdot\left(\sin \left(\beta+\theta_{5}\right)\right)}{\overline{O_{5} O_{4}^{\prime}} \cdot \sin \left(\alpha+\theta_{5}\right)+\overline{O_{4} O_{4}^{\prime}} \cdot \cos \left(\alpha+\theta_{5}\right)}
\end{aligned}
$$

where $F_{\Delta s}^{\mathrm{h}}, F_{\Delta s}^{\mathrm{v}}, F_{4}^{\mathrm{h}}$ and $F_{4}^{\mathrm{v}}$ are the horizontal and the vertical components of $F_{\Delta s}$ and $F_{4}$ (refer to Fig. $4 \mathrm{~b}$ ). The force $F_{4}$ generates a torque $T_{2}$ on the reel through the cam. $T_{2}$ is directly balanced by the cable force $F_{\Delta l}$. Equation 11 shows the relation between the cable force $F_{\Delta l}$ and $F_{4}$ :

$$
\begin{aligned}
T_{2}= & F_{4} \cdot \sin (\alpha) \overline{O_{3} C} \cdot \cos \left(\theta_{3}\right)+F_{4} \cdot \cos (\alpha) \overline{O_{3} C} \\
& \times \sin \left(\theta_{3}\right)=F_{\Delta l} R_{2} \\
F_{\Delta l}= & \frac{F_{4} \cdot \overline{O_{3} C} \cdot\left(\sin \left(\alpha+\theta_{3}\right)\right)}{R_{2}}
\end{aligned}
$$

Finally, by combining Eqs. 10 and 11 it is possible to find the relation between $F_{\Delta l}$ and $F_{\Delta s}$ that is:

$$
\begin{aligned}
F_{\Delta l}= & \frac{\overline{O_{3} C} \cdot\left(\sin \left(\alpha+\theta_{3}\right)\right)}{R_{2}} \cdot \\
& \times \frac{F_{\Delta s} \cdot L_{5} \cdot\left(\sin \left(\beta+\theta_{5}\right)\right)}{\overline{O_{5} O_{4}^{\prime}} \cdot \sin \left(\alpha+\theta_{5}\right)+\overline{O_{4} O_{4}^{\prime}} \cdot \cos \left(\alpha+\theta_{5}\right)}
\end{aligned}
$$

Using these relations, all the constructive parameters (e.g., reel and cam radius, linear tension spring stiffness, pivots location on the frame) of the non-linear spring were defined to meet the two design requirements stated above. 


\subsection{Muscles to joints}

The design of the linear elastic actuators described above is such that a non-linear force versus length relationship is established in which the position of the piston determines the rest length of the non-linear spring whilst the difference between the actual length and the rest length determines the force output. The non-linear mechanism acts such that the stiffness of the actuator $(\Delta F / \Delta l)$ increases monotonically with the amount of force. At the level of the individual actuators, there is no independent control of force and stiffness.

When two actuation units are coupled to act around a single joint, the opposing torques generated by each elastic element will result in a convergent torque field around a virtual EP $\theta_{\text {eq }}$ (i.e., the static position that the arm would reach if no external loads are applied). The equilibrium point as well as the torque field slope (i.e., joint stiffness) will be directly determined by the positions of the two hydraulic pistons. By properly moving the pistons the joint equilibrium point and stiffness change. To show how it is possible to independently adjust the EP and the joint stiffness, a mathematical description of the actuation scheme working principle will be reported hereafter.

According to Fig. 3, we define the initial configuration of the actuation system for the two joints. In this configuration, the non-linear elastic elements are unloaded, therefore the tendon cable forces are zero. The initial configuration of the shoulder angle $\left(\theta_{0}\right)$ is set to half the operative range of the joint, which becomes $\left[-90^{\circ},+90^{\circ}\right]$. Similarly, the initial configuration of pistons $\left(x_{\mathrm{ext}}^{0}\right.$ and $\left.x_{\mathrm{flx}}^{0}\right)$ are set to half the hydraulic piston movement range.

If we move the two pistons from the initial configuration in two arbitrary positions $x_{\text {ext }}$ and $x_{\mathrm{flx}}$, the two transmission lines will be deformed respectively of $\Delta l_{\text {ext }}$ and $\Delta l_{\mathrm{flx}}$, depending on the joint angle $\theta$ :

$$
\begin{aligned}
\Delta l_{\mathrm{ext}} & =\left(x_{\mathrm{ext}}^{0}-x_{\mathrm{ext}}\right) a_{\mathrm{f}}+R\left(\theta-\theta_{0}\right) \\
& =\Delta x_{\mathrm{ext}} a_{\mathrm{f}}+R\left(\theta-\theta_{0}\right) \\
\Delta l_{\mathrm{flx}} & =\left(x_{\mathrm{flx}}^{0}-x_{\mathrm{flx}}\right) a_{\mathrm{f}}-R\left(\theta-\theta_{0}\right) \\
& =\Delta x_{\mathrm{flx}} a_{\mathrm{f}}-R\left(\theta-\theta_{0}\right)
\end{aligned}
$$

that assuming $\theta_{0}=0$ become:

$$
\begin{aligned}
\Delta l_{\mathrm{ext}} & =\Delta x_{\mathrm{ext}} a_{\mathrm{f}}+R \theta \\
\Delta l_{\mathrm{flx}} & =\Delta x_{\mathrm{flx}} a_{\mathrm{f}}-R \theta
\end{aligned}
$$

where $R$ is the radius of the NEURARM shoulder joint pulley, $\Delta x_{\mathrm{ext}}=x_{\mathrm{ext}}^{0}-x_{\mathrm{ext}}$ and $\Delta x_{\mathrm{flx}}=x_{\mathrm{flx}}^{0}-x_{\mathrm{flx}}$. Assuming the steel cable infinitively stiff, all the transmission line deformation will be that of the non-linear elastic element. So, the antagonistic cables act on the shoulder joint with two forces $F_{\text {ext }}\left(\Delta l_{\text {ext }}\right)$ and $F_{\text {flx }}\left(\Delta l_{\text {ffx }}\right)$ :

$$
\begin{aligned}
F_{\mathrm{ext}} & =a_{1} \Delta l_{\mathrm{ext}}^{2}+a_{2} \Delta l_{\mathrm{ext}} \\
F_{\mathrm{flx}} & =a_{1} \Delta l_{\mathrm{flx}}^{2}+a_{2} \Delta l_{\mathrm{flx}}
\end{aligned}
$$

Assuming the flexor unit responsible for a positive torque, the resulting torque $T$ applied on the joint is consequently equal to:

$T=R\left(F_{\text {flx }}-F_{\text {ext }}\right)$

The joint equilibrium angle $\theta_{\mathrm{eq}}$ is easily calculated solving with respect to $\theta$ the equation $T=0 \Rightarrow F_{\mathrm{flx}}=F_{\mathrm{ext}} \Rightarrow$ $\Delta l_{\mathrm{flx}}=\Delta l_{\mathrm{ext}}$ :

$\Delta_{\mathrm{flx}}=\Delta_{\mathrm{ext}} \Rightarrow \theta_{\mathrm{eq}}=\frac{a_{\mathrm{f}}\left(\Delta x_{\mathrm{flx}}-\Delta x_{\mathrm{ext}}\right)}{2 R}$

By appropriately changing the reference frames for the piston positions, it is possible to have $x_{\mathrm{flx}}^{0}=x_{\mathrm{ext}}^{0}=0$, so that (16) becomes:

$\theta_{\mathrm{eq}}=\frac{a_{\mathrm{f}}\left(x_{\mathrm{ext}}-x_{\mathrm{ffx}}\right)}{2 R}$

Substituting (14) and (15) in the Eq. 16 and differentiating with respect to the joint angle, it is possible to find the joint stiffness $K_{\theta}$ :

$$
\begin{aligned}
K_{\theta}= & -\frac{\partial T}{\partial \theta}=-\frac{\partial}{\partial \theta}\left(R\left(F_{\mathrm{flx}}-F_{\mathrm{ext}}\right)\right)=-2 R^{2} \\
& \times\left(a_{2}-a_{1} a_{\mathrm{f}}\left(x_{\mathrm{ext}}+x_{\mathrm{flx}}\right)\right)
\end{aligned}
$$

As Eqs. 18 and 19 show, the joint position is proportional to the difference between the two piston positions $\left(x_{\mathrm{ext}}-x_{\mathrm{ext}}\right)$, whilst the joint stiffness depends on their sum $\left(x_{\mathrm{ext}}+x_{\mathrm{ext}}\right)$. Thereby, the joint position and stiffness can be regulated independently. This can be better shown by introducing two new control variables as a linear combination of the two piston positions. The first, the differential command, is defined as a reciprocal shift of the antagonist pistons:

$x_{\mathrm{dif}}=\frac{\left(x_{\mathrm{ext}}-x_{\mathrm{flx}}\right)}{2}$

The second is the common mode command, which is an equal shift of the antagonist pistons:

$x_{\mathrm{com}}=-\frac{\left(x_{\mathrm{ext}}+x_{\mathrm{flx}}\right)}{2}$

Substituting Eqs. 20 and 21 into 18 and 19, we get the final equations describing the joint position (22) and stiffness (23):

$\theta_{\mathrm{eq}}=\frac{a_{\mathrm{f}} x_{\mathrm{dif}}}{R}$

$K_{\theta}=2 R^{2}\left(a_{2}+2 a_{1} a_{\mathrm{f}} x_{\mathrm{com}}\right)$

As stated above, the elbow joint presents a more complex structure compared to the shoulder. Indeed, the elbow flexor moment arm changes significantly as a non-linear function of the joint angular position, whilst the extensor moment arm can be considered to remain relatively constant (Fig. 3b). 
Consequently, the mathematical model proposed for the shoulder joint must be modified to describe the elbow joint working principle. Because of the variable flexor moment arm, it is not possible to define an equal common mode $\left(x_{\mathrm{com}}\right)$ and differential mode command $\left(x_{\mathrm{dif}}\right)$ for the flexor and the extensor units. As a result, the two elbow piston positions are defined as:

$x_{\mathrm{flx}}=-x_{\mathrm{com}_{-} \mathrm{flx}}-x_{\mathrm{dif}_{f} \mathrm{flx}}$

$x_{\text {ext }}=-x_{\text {com_ext }}+x_{\text {dif_ext }}$

The initial configuration of the elbow joint $\left(\theta_{0}\right)$ is equal to the fully extended joint position, defining a joint angular range $\left[0^{\circ}, 140^{\circ}\right]$. Accordingly, the initial configuration of the flexor piston $\left(x_{\mathrm{flx}}^{0}\right)$ corresponds to the fully extended position, whilst the extensor one $\left(x_{\mathrm{ext}}^{0}\right)$ is fully retracted. The lengths of the elbow-joint actuation units $\left(\Delta l_{\mathrm{ffx}}, \Delta l_{\mathrm{ext}}\right)$ change as a function of the joint angle $(\theta)$ and the two piston positions $\left(x_{\mathrm{flx}}, x_{\mathrm{ext}}\right)$ :

$$
\begin{aligned}
\Delta l_{\mathrm{flx}}= & \left(x_{\mathrm{flx}}^{0}-x_{\mathrm{flx}}\right) a_{\mathrm{f}}-I_{\mathrm{ffx}}(\theta)=\Delta x_{\mathrm{flx}} a_{\mathrm{f}}-I_{\mathrm{flx}}(\theta) \\
= & -x_{\mathrm{flx}} a_{\mathrm{f}}-I_{\mathrm{flx}}(\theta) \\
\Delta l_{\mathrm{ext}}= & \left(x_{\mathrm{ext}}^{0}-x_{\mathrm{ext}}\right) a_{\mathrm{f}}+R\left(\theta-\theta_{0}\right)=\Delta x_{\mathrm{ext}} a_{\mathrm{f}} \\
& +R\left(\theta-\theta_{0}\right)=-x_{\mathrm{ext}} a_{\mathrm{f}}+R \theta
\end{aligned}
$$

where $I_{\mathrm{flx}}(\theta)$ is the integral of the variable flexor moment arm, $R_{\mathrm{flx}}(\theta)$, along the joint angular position that is:

$I_{\mathrm{flx}}(\theta)=\int_{\theta_{0}}^{\theta} R_{\mathrm{flx}}(\omega) \mathrm{d} \omega=\int_{0}^{\theta} R_{\mathrm{flx}}(\omega) \mathrm{d} \omega$

The joint torque static equilibrium equation is described as:

$$
\begin{aligned}
T_{\text {elbow }} & =0 \Rightarrow T_{\mathrm{flx}}=T_{\mathrm{ext}} \Rightarrow R_{\mathrm{flx}}(\theta) F\left(\Delta l_{\mathrm{flx}}\right) \\
& =R_{\mathrm{ext}} F\left(\Delta l_{\mathrm{ext}}\right)
\end{aligned}
$$

Note that because of the difference between the two moment $\operatorname{arms}\left(R_{\mathrm{flx}}(\theta), R_{\mathrm{ext}}\right)$, the equal forces condition (i.e., $F_{\mathrm{flx}}=$ $F_{\text {ext }}$ ) does not guarantee joint equilibrium. Nevertheless, if we assume both the actuation units have been unloaded, it is possible to solve the static equilibrium equation independent of the moment arms:

$$
\left.\begin{array}{l}
\Delta l_{\text {flx }}=0 \Rightarrow F\left(\Delta l_{\text {flx }}\right)=0 \\
\Delta l_{\text {ext }}=0 \Rightarrow F\left(\Delta l_{\text {ext }}\right)=0
\end{array}\right\} \Rightarrow T_{\text {elbow }}=0
$$

By applying (29) to the elongation Eq. 26, the flexor and extensor differential commands ( $x_{\text {dif_flx }}$ and $\left.x_{\text {dif_ext }}\right)$ can be evaluated as a function of the sole angular $\operatorname{EP}\left(\theta_{\mathrm{eq}}\right)$ :

$$
\begin{aligned}
& x_{\text {dif_ext }}=\frac{R_{\text {ext }} \theta_{\text {eq }}}{a_{\mathrm{f}}} \\
& x_{\text {dif_flx }}=\frac{I_{\text {flx }}\left(\theta_{\text {eq }}\right)}{a_{\mathrm{f}}}
\end{aligned}
$$

As introduced by Eqs. 24 and 25, the elbow joint stiffness regulation requires two different common mode commands $\left(x_{\text {com_flx }}\right.$ and $\left.x_{\text {com_ext }}\right)$. Indeed, because of the difference between the two moment arms $\left(R_{\mathrm{flx}}(\theta), R_{\mathrm{ext}}\right)$, the flexor and extensor torque/elongation curves $\left(T_{\mathrm{flx}}(\Delta l), T_{\mathrm{ext}}(\Delta l)\right)$ differ even if the force/elongation characteristics are identical $\left(F_{\text {flx }}(\Delta l)=F_{\text {ext }}(\Delta l)\right)$.

The common mode commands specify the torque generated by the two antagonist cables by imposing two different elongations so that:

- the stiffness can be regulated by exploiting the nonlinearity of the force/elongation curves;

- the joint EP remains unchanged (i.e., Eq. 28 is satisfied).

The resulting elbow joint stiffness is a function of both the angular position and the common mode commands:

$$
\begin{aligned}
K & \left(\theta, x_{\text {com_ext }}, x_{\text {com_flx }}\right) \\
= & \frac{\mathrm{d} T}{d \theta} \\
= & \frac{\mathrm{d}}{\mathrm{d} \theta}\left(R_{\mathrm{flx}}(\theta) F\left(\Delta l_{\mathrm{ffx}}\right)-R_{\mathrm{ext}} F\left(\Delta l_{\mathrm{ext}}\right)\right) \\
= & \frac{\mathrm{d}}{\mathrm{d} \theta}\left(\begin{array}{c}
R_{\mathrm{flx}}(\theta)\left(p_{1}\left(-x_{\mathrm{com} \_\mathrm{fl}} a_{\mathrm{f}}-I_{\mathrm{ffx}}(\theta)\right)^{2}\right. \\
\left.+p_{2}\left(-x_{\text {com_flx }} a_{\mathrm{f}}-I_{\mathrm{ffx}}(\theta)\right)\right) \\
-R_{\text {ext }}\left(-x_{\text {com_ext }} a_{\mathrm{f}}+R \theta\right)^{2} \\
+p_{2}\left(-x_{\text {com_ext }} a_{\mathrm{f}}+R \theta\right)
\end{array}\right)
\end{aligned}
$$

Moreover, by applying the torque equilibrium condition (28) to the cable elongation equations, the relation between the two common mode commands can be evaluated as:

$$
\begin{aligned}
& R_{\mathrm{flx}}\left(\theta_{\mathrm{eq}}\right)\left[p_{1}\left(a_{\mathrm{f}} x_{\mathrm{com} \_\mathrm{fxx}}\right)^{2}+p_{2} a_{\mathrm{f}} x_{\mathrm{com} \_\mathrm{ftx}}\right] \\
& =R\left[p_{1}\left(a_{\mathrm{f}} x_{\mathrm{com} \_\mathrm{ext}}\right)^{2}+p_{2} a_{\mathrm{f}} x_{\mathrm{com} \_\mathrm{ext}}\right]
\end{aligned}
$$

Thus, $x_{\text {com_flx }}$ can be expressed as a function of $x_{\text {com_ext }}$.

At the end, we used a numerical simulation to calculate the joint stiffness from the Eqs. 19 and 20. The simulation result produces a bi-dimensional plot of $K\left(\theta_{\text {eq }}, x_{\text {com_ext }}\right)$, illustrated in Fig. 5, that shows how joint stiffness varies as a function of the co-activation command and the joint angle at equilibrium. An important feature of this mapping is that joint stiffness increases smoothly and monotonically as $x_{\text {com_ext }}$ and $\theta_{\text {eq }}$ increase.

\subsection{Decoupling control of position and stiffness}

We developed a multi-layered control architecture to independently control the joint position and stiffness. The low level-layer controls the hydraulic piston position $\left(x_{\mathrm{ext}}, x_{\mathrm{flx}}\right)$, and is thus responsible for the regulation of the rest lengths of the actuation lines. This control has been detailed in Vitiello 


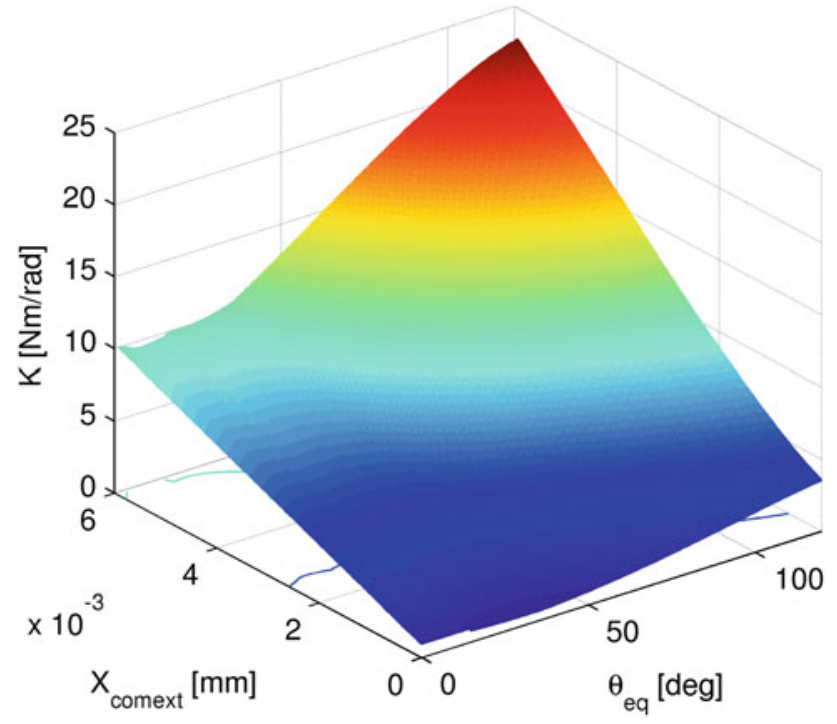

Fig. 5 Elbow joint stiffness as a function of the co-activation command $\left(x_{\text {com_ext }}\right)$ and the angular position $\left(\theta_{\text {eq }}\right)$

et al. (2007). The high level controller is dedicated to the coordination of the piston positions. For each joint it determines the two desired actuation unit rest lengths $\left(x_{\mathrm{flx}}^{\mathrm{des}}, x_{\mathrm{ext}}^{\mathrm{des}}\right)$ as a function of the desired angular EP $\left(\theta_{\mathrm{eq}}^{\mathrm{des}}\right)$ and stiffness level $\left(K_{\mathrm{eq}}^{\mathrm{des}}\right)$. As illustrated in Fig. 6, the desired angular position and stiffness are input variables of the high level control of the shoulder joint, and are separately processed. The desired EP determines a reciprocal shift of the pistons ( $\left.x_{\mathrm{dif}}\right)$ by inverting Eq. 22:

$x_{\mathrm{dif}}=\frac{R \theta_{\mathrm{eq}}}{a_{\mathrm{f}}}$
The desired joint stiffness determines the equal shift of the pistons $\left(x_{\text {com }}\right)$, by Eq. 23:

$x_{\mathrm{com}}=\frac{K_{\theta}-2 a_{2} R^{2}}{4 a_{1} a_{\mathrm{f}} R^{2}}$

Finally, $x_{\text {dif }}$ and $x_{\text {com }}$ lead to the desired piston positions for both the flexor and the extensor unit $\left(x_{\mathrm{flx}}^{\mathrm{des}}, x_{\mathrm{ext}}^{\mathrm{des}}\right)$ by means of Eqs. 22 and 23.

The elbow joint high-level controller differs from that of the shoulder. The desired EP determines the differential commands $\left(x_{\text {dif_flx }}\right.$ and $\left.x_{\text {dif_ext }}\right)$ by means of Eq. 30 . Then, both the desired EP and the desired joint stiffness participate to the definition of the common mode commands. First, the extensor common mode command $\left(x_{\text {com_ext }}\right)$ is computed by means of the numerical resolution of Eq. 31 . Then, the flexor common mode command $\left(x_{\text {com_flx }}\right)$ is calculated from Eq. 32.

It is important to remark that this controller acts in an openloop fashion. Therefore, the resulting static and dynamic performances are only related to the 'hardware' viscoelastic behaviour of the actuation, even if the mechanical behaviour can be regulated through the controller.

\section{Experiments I: performance versus design goals at the joint level}

\subsection{Muscle-like actuators}

The non-linear element mathematical model introduced in the previous Section was numerically simulated to verify that all the design requirements were met and to test whether the NEURARM actuators captures the salient features of

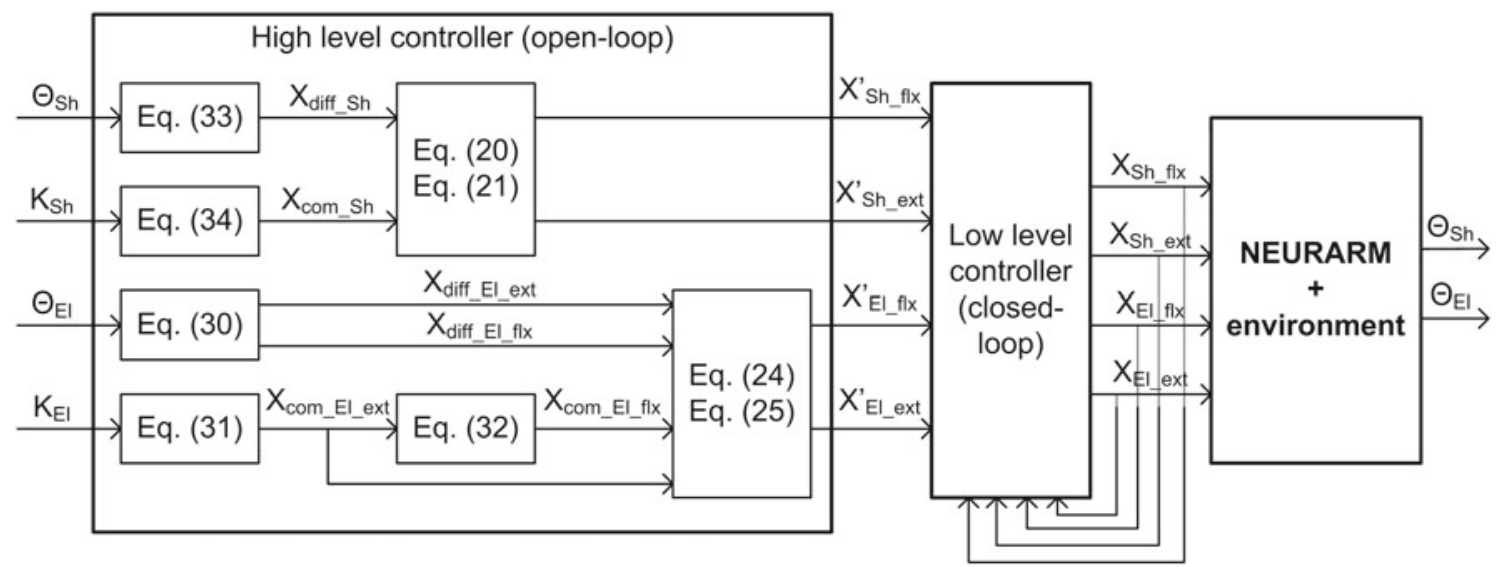

Fig. 6 The multi-layered NEURARM open-loop position and stiffness controller: the high level controller takes as input the desired EPs and the desired joints stiffness, and puts on output, for each joint, the flexor and extensor virtual piston positions; the virtual positions are then the references for the low level controller, which is a closed-loop position regulator; finally, the interaction between the NEURARM intrinsic mechanical properties and the external environment determines the joints EPs 


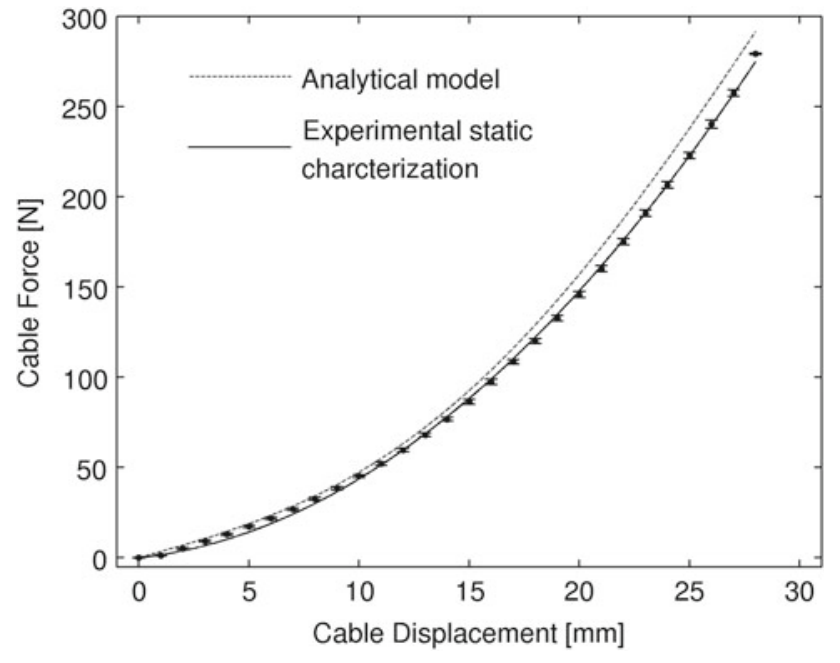

Fig. 7 Non-linear element analytical model simulation versus experimental characterization

muscles as they apply to the hypotheses of impedance control. We first characterized the length-tension characteristics of a single actuator complex. The steel cable exploited for the NEURARM actuation has a length of about $50 \mathrm{~cm}$, resulting in an equivalent elastic constant of about $100 \mathrm{~N} / \mathrm{mm}$, which could not be actually neglected in the simulation. The elastic characteristics of the steel cable were therefore modelled by introducing an additional virtual spring (i.e., a constant stiffness element) in series with the non-linear element model.

The simulation results are illustrated in Fig. 7. The resulting non-linear force/elongation curve was well approximated by a second order polynomial function with coefficients $a_{1}=3.326 \cdot 10^{5} \mathrm{Nm}^{-2}$ and $a_{2}=1009 \mathrm{Nm}^{-1}$, giving a root-mean-square error (RMSE) value equal to $1.70 \mathrm{~N}$. These coefficients, according to Eqs. 7 and 21, result in a theoretical shoulder and elbow stiffness in the range $K_{\mathrm{Sh}}=$ $[2.5-40] \mathrm{Nm} / \mathrm{rad}$, thus satisfying the design requirements. Furthermore, the form of the torque/displacement curve is such that angular stiffness increases with torque, which is the key factor that allows for stiffness modulation via co-contraction of opposing muscles.

Note that the maximum achievable stiffness is limited by the maximum cable loading force (about $400 \mathrm{~N}$ ), as determined by the structural strength of the non-linear element. According to the force versus deformation characteristic of the non-linear element (Eq. 1), the maximum force value is gathered at a deformation of $0.034 \mathrm{~m}$, meaning that it is achieved by setting $x_{\text {com }}$ equal to $0.0086 \mathrm{~m}$ (thanks to the stroke amplifier that multiplies by a factor of four the piston displacement).

In a previous study (Vitiello et al. 2008), the non-linear element force $\left(F_{\Delta l}\right)$ versus elongation $(\Delta l)$ curve was experimentally characterized, both in static and dynamic conditions. The static experimental curves were fitted by a second order polynomial function, giving as a result the coefficients $a_{1}=3.145 \cdot 10^{5} \mathrm{Nm}^{-2}$ and $a_{2}=935 \mathrm{Nm}^{-1}$, and a RMSE of $1.62 \mathrm{~N}$. In addition, the dynamic characterization, performed with elongation velocities up to $400 \mathrm{~mm} / \mathrm{min}$, demonstrated a maximum hysteresis of $18.3 \mathrm{~N}$ corresponding to the $6 \%$ of the force exerted at maximum spring elongation.

The comparison between the numerical simulation and the experimental characterization curves is shown in Fig. 7. The maximum force difference is $16.6 \mathrm{~N}$ which corresponds to $5 \%$ of the non-linear element force limit. The good agreement between the mathematical model and the prototype validates both the design of the non-linear element and its technological implementation.

\subsection{Static characterization of a single joint}

As introduced in Sect. 2, the antagonistic actuation system acting in combination with the rest-length controllers on the non-linear actuators is intended to generate a convergent torque field around an EP of the joint. Once the EP is set by an appropriate differential command $\left(x_{\mathrm{dif}}\right)$, the torque field should be generated and maintained by the intrinsic properties of the actuation system. Moreover, the controller should be able to modify the mechanical behaviour of the joint by acting on the slope of the torque field (i.e., joint stiffness) through the common mode command. In order to verify experimentally that the NEURARM can fulfil this requirement, the following procedure was applied for the shoulder and the elbow separately:

1. The desired EP was set by the appropriate differential command $\left(x_{\mathrm{dif}}\right)$.

2. The joint intrinsic stiffness was adjusted by the common mode command $\left(x_{\text {com }}\right)$.

3. While recording joint torques, we applied quasi-static displacements of the NEURARM joint in both positive and negative directions. The absolute value of the maximum displacement in either direction was $10^{\circ}$. The displacement was manually applied by means of a passive mechanism that interfaces the NEURARM end-effector through 6-axis load cell, which provided an independent measurement of the resulting joint torque.

Five different joint stiffness levels were tested for each joint to prove that the system is capable of significant modulation of the passive joint stiffness. The characterization procedure was repeated ten times for each joint stiffness level. During the characterization of the elbow, the shoulder was blocked mechanically and vice-versa.

Note that, for the characterization experiments, we inten-

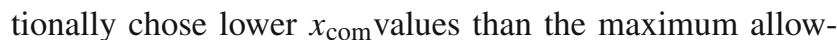
able one $(0.0086 \mathrm{~mm})$. This way, we kept low the pre-loading force on the non-linear elements, thus avoiding exceeding 

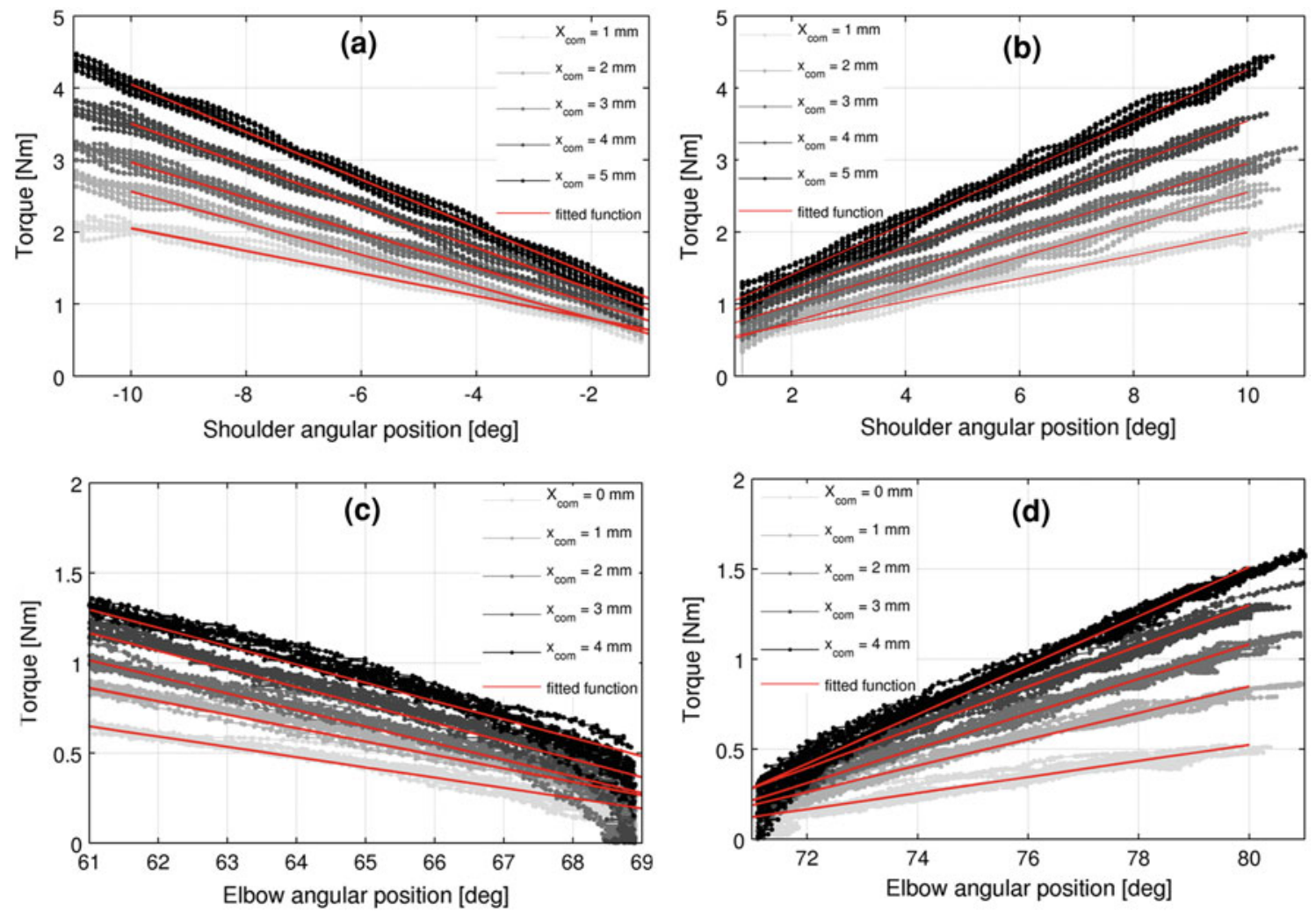

Fig. 8 Single joint static characterization results: (a) shoulder joint perturbed in extension, (b) shoulder joint perturbed in flexion, (c) elbow joint perturbed in extension and (d) elbow joint perturbed in flexion

the maximum loading force whilst displacing the arm from its EP.

The resulting torque/displacement curves are reported in Fig. 8a for the shoulder and in Fig. 8b for the elbow. The slope of the torque fields was then calculated by fitting the torque/displacement curves with a linear function for positive and negative displacement independently. The fitting results, reported in Table 1, confirm the numerical simulation outputs reported in Sect. 3.2: the measured stiffness values corresponded well to the theoretical value, giving a RMSE in the range of $0.17-0.055 \mathrm{Nm}$. This small error can be attributed to friction that is mainly generated in Bowden cables (Schiele et al. 2006). It is worth noting that friction is low and does not affect much the overall system performance. Future studies could aim at reducing this effect through modification of the Bowden cables structure.

Table 1 Single joint static characterization, linear fitting results

\begin{tabular}{|c|c|c|c|c|}
\hline \multicolumn{2}{|l|}{ Input } & \multicolumn{3}{|l|}{ Fitting output } \\
\hline \multirow[b]{2}{*}{$x_{\text {com }}(\mathrm{mm})$} & \multirow[b]{2}{*}{$K(\mathrm{Nm} / \mathrm{rad})$} & \multicolumn{2}{|l|}{ Slope $(\mathrm{Nm} / \mathrm{rad})$} & \multirow[t]{2}{*}{ RMSE (Nm) } \\
\hline & & Positive displacement & Negative displacement & \\
\hline \multicolumn{5}{|c|}{ Shoulder joint static characterization-fitting results } \\
\hline 1 & 6.589 & 9.110 & 9.282 & 0.055 \\
\hline 2 & 10.199 & 12.433 & 12.777 & 0.051 \\
\hline 3 & 14.037 & 13.866 & 14.209 & 0.055 \\
\hline 4 & 17.533 & 16.501 & 16.788 & 0.049 \\
\hline 5 & 21.199 & 19.08 & 20.397 & 0.052 \\
\hline \multicolumn{5}{|c|}{ Elbow joint static characterization-fitting results } \\
\hline 0 & 1.776 & 3.552 & 2.406 & 0.051 \\
\hline 1 & 4.068 & 4.240 & 4.011 & 0.017 \\
\hline 2 & 5.672 & 4.927 & 5.271 & 0.020 \\
\hline 3 & 6.818 & 5.443 & 6.073 & 0.022 \\
\hline 4 & 8.422 & 5.787 & 7.047 & 0.026 \\
\hline
\end{tabular}


Fig. 9 Shoulder joint step responses for different stiffness levels (i.e., $K=6.589,10.199$, $14.037,17.533$ and $21.199 \mathrm{Nm} / \mathrm{rad}$ ) and different elbow joint positions; (a) shoulder moves in flexion, elbow is fixed in maximum extension,

(b) shoulder moves in flexion, elbow is fixed in maximum flexion, (c) shoulder moves in extension, elbow is fixed in maximum extension and (d) shoulder moves in extension, elbow is fixed in maximum flexion

Fig. 10 Elbow joint step responses for different joint stiffness levels $(K=4.068$, $5.672,6.818$ and $8.422 \mathrm{Nm} / \mathrm{rad})$ and different movement direction: (a) elbow moves in flexion and (b) elbow moves in extension
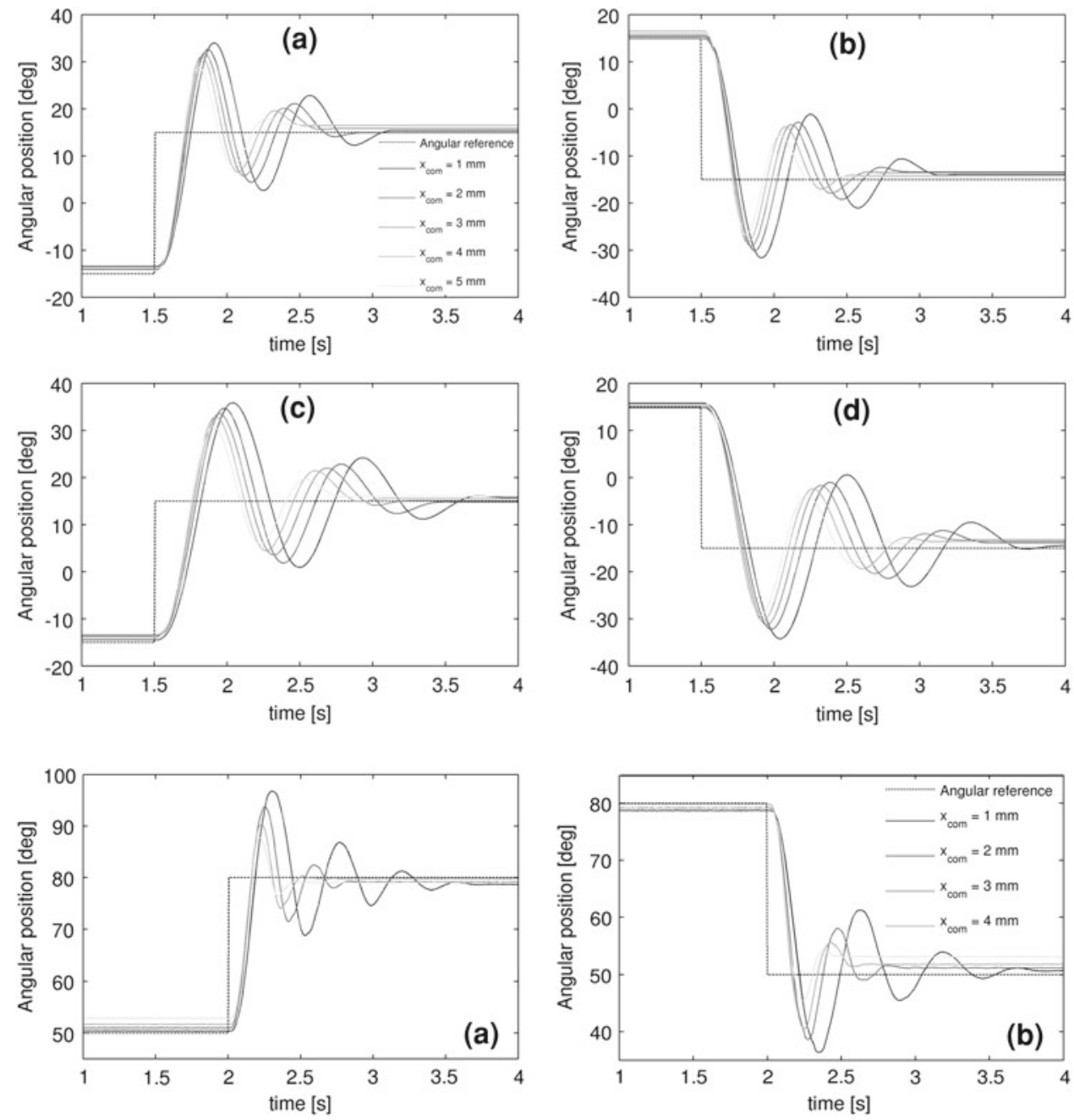

\subsection{Dynamic characterization of a single joint}

According to the principles of impedance control, the elastic properties of the neuromuscular system are important not only for posture maintenance but also to generate movements (Bizzi et al. 1984). In fact, by modifying the joint mechanical behaviour (i.e., joint stiffness), it should be possible to affect the system dynamic response. For this reason, the NEURARM actuation was designed to allow the joint stiffness regulation to be independent of the position control. In order to show that the NEURARM is able to vary its mechanical behaviour in dynamic tasks, a step response characterization was performed with several levels of joint stiffness. The resulting trajectories were then used to model the joint as a second-order system, to properly quantify the changes in the dynamic behaviour. Finally, the joint transfer function was estimated for each stiffness level by fitting the response of the platform to multiple sine wave inputs.

The step response of the joint-position controller was recorded for different stiffness levels. A step of $30^{\circ}$ of amplitude was chosen as desired joint angle. For each stiffness level, 50 consecutive cycles of two steps, from 15 to $-15^{\circ}$ and vice versa for the shoulder and from 50 to $80^{\circ}$ and vice versa for the elbow were performed. Each joint is mechanically locked, in turn, whilst the other is being characterized. The shoulder characterization was performed with the elbow fixed in two different positions: at maximum flexion (i.e., $0^{\circ}$ ) and at maximum extension (i.e., $140^{\circ}$ ), to test the effect of an increased joint inertia.

The step response results are shown in Fig. 9 for the shoulder and Fig. 10 for the elbow. For each stiffness level, the angular trajectory averaged over 50 cycles has been reported. The averaged trajectories are compared with the desired angular position.

We carried out a quantitative analysis of the step responses by fitting the NEURARM joint response with a secondorder model. The fitting results show low RMSE values, in the range: $1.67 \pm 0.26^{\circ}$. Tables 2 and 3 report the estimated natural frequencies, the damping factors and the fitting RMSE for the shoulder and the elbow, respectively. The 
Table 2 Shoulder joint step responses analysis: the angular step responses are fitted with an ideal second order model

\begin{tabular}{|c|c|c|c|c|c|c|c|}
\hline \multicolumn{8}{|c|}{ Shoulder joint } \\
\hline \multirow{2}{*}{\multicolumn{2}{|c|}{ Input }} & \multicolumn{6}{|l|}{ Fitting Output } \\
\hline & & \multicolumn{3}{|c|}{ Elbow in maximum extension } & \multicolumn{3}{|c|}{ Elbow in maximum flexion } \\
\hline$x_{\mathrm{com}}(\mathrm{mm})$ & $K(\mathrm{Nm} / \mathrm{rad})$ & Natural frequency $(\mathrm{Hz})$ & Damping factor & $\operatorname{RMSE}\left({ }^{\circ}\right)$ & Natural frequency $(\mathrm{Hz})$ & Damping factor & $\operatorname{RMSE}\left(^{\circ}\right)$ \\
\hline 1 & 6.589 & 1.100 & 0.138 & 1.611 & 1.492 & 0.142 & 1.504 \\
\hline 2 & 10.199 & 1.236 & 0.145 & 1.516 & 1.678 & 0.149 & 1.451 \\
\hline 3 & 14.037 & 1.335 & 0.146 & 1.641 & 1.820 & 0.158 & 1.554 \\
\hline 4 & 17.533 & 1.441 & 0.156 & 1.602 & 1.983 & 0.168 & 1.517 \\
\hline 5 & 21.199 & 1.535 & 0.173 & 1.420 & 2.132 & 0.192 & 1.397 \\
\hline
\end{tabular}

The resulting natural frequency and damping factor are reported, along with the fitting RMSE, as a function of the joint stiffness level and elbow joint position fixed during the trials

Table 3 Elbow joint step responses analysis: the angular step responses are fitted with an ideal second order model; the resulting natural frequency and damping factor are reported, along with the fitting RMSE, as a function of the joint stiffness level

\begin{tabular}{|c|c|c|c|c|}
\hline \multicolumn{5}{|l|}{ Elbow joint } \\
\hline \multicolumn{2}{|l|}{ Input } & \multicolumn{3}{|l|}{ Fitting output } \\
\hline$x_{\text {com }}(\mathrm{mm})$ & $K(\mathrm{Nm} / \mathrm{rad})$ & Natural frequency $(\mathrm{Hz})$ & Damping factor & $\operatorname{RMSE}\left({ }^{\circ}\right)$ \\
\hline 1 & 4.068 & 1.935 & 0.173 & 2.115 \\
\hline 2 & 5.672 & 2.681 & 0.187 & 1.956 \\
\hline 3 & 6.818 & 3.055 & 0.203 & 1.915 \\
\hline 4 & 8.422 & 3.339 & 0.247 & 2.216 \\
\hline
\end{tabular}

natural frequency increases proportionally to the joint stiffness level, in agreement with the ideal system response (i.e., $f_{0}=\frac{\omega_{n}}{2 \pi}=\frac{1}{2 \pi} \sqrt{\frac{K}{I}}$ ). The damping factor (i.e., $\xi=\frac{B}{2 \sqrt{K I}}$ ) increases with the stiffness level too. This latter effect results from the simultaneous increase of the stiffness $(K)$ and the damping $(B)$. As a matter of fact, the damping is not a controlled variable, but it increases proportionally to the stiffness. Indeed, the friction inside the Bowden cable grows with the preloading force increment, which is needed to increase the stiffness. Nevertheless, the fact is that the damping in biological systems also tends to increase with muscle force (Cannon and Zahalak 1982). So, although this 'feature' of the mechanical actuator system was not intentionally included by design, it does further the resemblance between the robotic arm and natural biological systems. Finally, the effect of the inertia is consistent with the fitting model since both the natural frequency and the damping factor decrease when the stiffness is increased.

In order to verify the reliability of the fitting results obtained by the second-order model, we performed transfer function estimation by inputting multiple sine-wave references to the controller. For each joint and for each stiffness level (the same used in the step response trials), different sine waves were applied with a constant amplitude of $30^{\circ}$ and an angular offset of $0^{\circ}$ for the shoulder and $65^{\circ}$ for the elbow. The resulting angular trajectories, reported in Fig. 11, were used for estimating the transfer functions of the system. The natural frequencies, corresponding to the resonance peaks of the transfer functions estimated by the multi-sine inputs, perfectly agree with that estimated by the step response methods. This further confirms that NEURARM control can modulate its joint mechanical behaviour in dynamic tasks.

\section{Experiments II: global (endpoint) characteristics compared to human performance}

Having designed, implemented and tested the joint-level characteristics of the NEURARM, we then set out to test to what extent these features alone can account for known behaviour of the human arm. We chose at this stage to quantify the quasi-static behaviour of the limb in terms of the endpoint stiffness and the dynamic behaviour of the limb in terms of trajectory following in point-to-point movements and compared these observations to data on human movements from the literature.

\subsection{Endpoint static behaviour (Cartesian stiffness)}

The static analysis of the single joint mechanical properties cannot be directly extended to the multi-joint posture tasks because of the non-linearity introduced by the kinematics of the arm. Indeed, the spring-like behaviour of the two-joint 

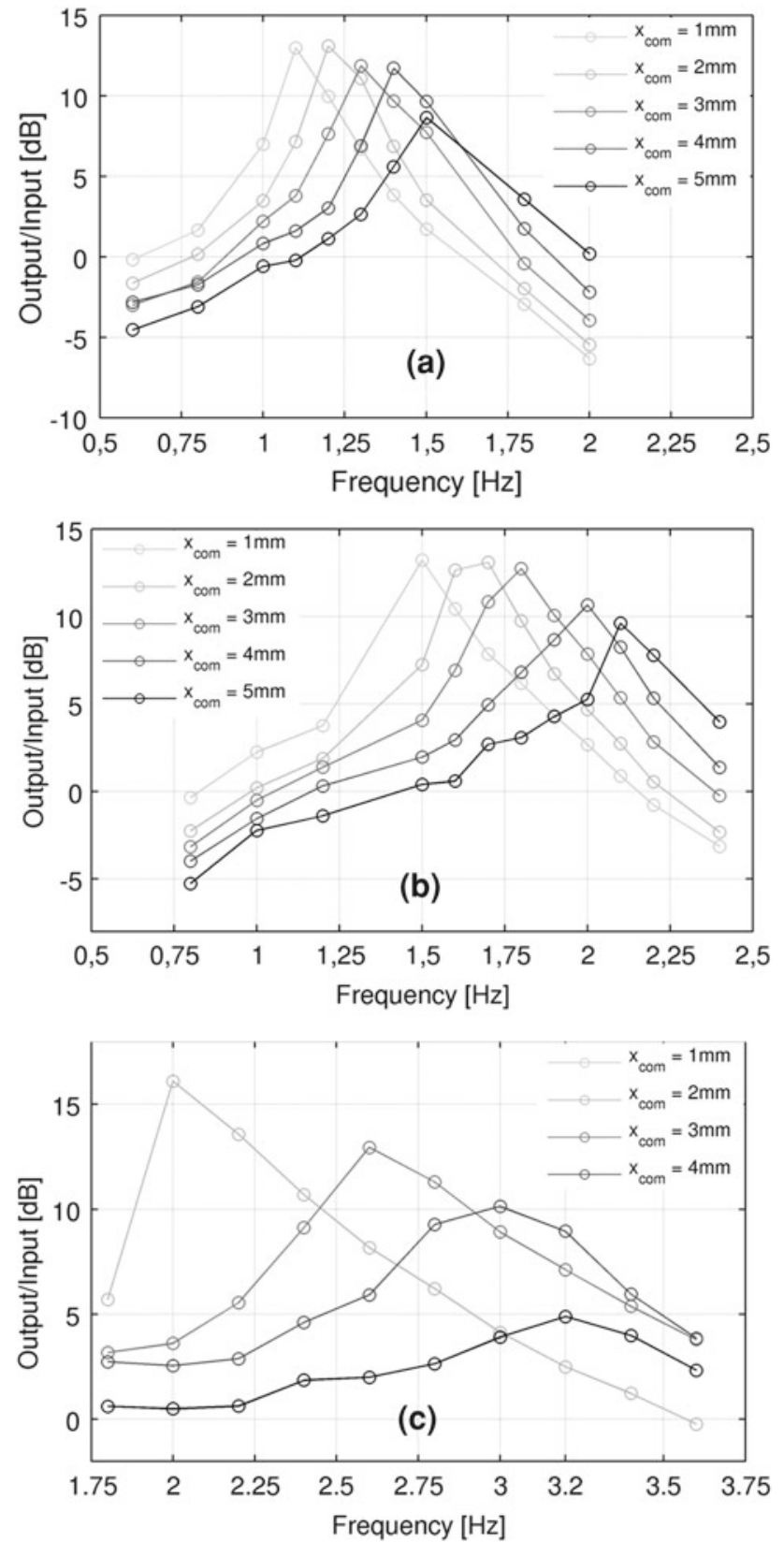

Fig. 11 Single joint transfer functions estimation by multiple-sine waves inputs; the shoulder joint transfer function is estimated for different stiffness levels (i.e., $K=6.589,10.199,14.037,17.533$ and $21.199 \mathrm{Nm} / \mathrm{rad}$ ), whilst keeping the elbow fixed in maximum extension position (a) and maximum flexion position (b); (c) the elbow joint transfer function is estimated for four different stiffness levels (i.e., $K=4.068,5.672,6.818,8.422 \mathrm{Nm} / \mathrm{rad}$ )

arm in Cartesian space is described by a stiffness matrix K, which depends on both the position of the joints and the joint stiffness, through the Jacobian matrix (i.e., $\mathrm{Kj}=\mathrm{J}^{T} \mathrm{KJ}$ ). Since the Jacobian matrix $\mathrm{J}$ varies as a function of joint position, the endpoint stiffness matrix varies in a complex fashion as a function of joint configuration, lever arms and actuator stiffnesses.
To empirically evaluate the endpoint stiffness of the NEURARM, we extended the static perturbation approach that we used for the single-joint static characterization to Cartesian space by applying a procedure similar to that used in the characterization of the human arm spring-like behaviour, as in Mussa-Ivaldi et al. (1985). Specifically, we applied displacements to the endpoint and recorded the corresponding static forces generated by the NEURARM linkage. The displacements were manually applied through a passive mechanism that interfaced to the NEURARM endeffector through a six-axis load cell and guided the movement towards the correct positions that should be applied for the characterization. This passive mechanism guaranteed high repeatability and accuracy, thus ensuring the reliability of the experiment. For each EP eight displacements with different directions were applied to have a reliable stiffness measurement. From the measured displacements and forces, the stiffness matrix was obtained by linear regression of the force/displacement vectors.

Five different end-effector EPs and four joint stiffness combinations were characterized, as reported in Table 4, to point out the similarity between the human Cartesian space behaviour and the NEURARM one. Moreover, Fig. 12 summarizes the results showing some stiffness ellipses derived following the procedure described by Mussa-Ivaldi et al. (1985). Figure 12a shows how the stiffness ellipsis is affected by a displacement of the joint positions whilst the joint stiffness level is kept constant, whereas Fig. 12b illustrates the effect of the joint stiffness adjustment in a fixed position. The three parameters characterizing the stiffness ellipses (i.e., size, orientation and shape) are reported in Table 5 for all the measured stiffness matrices. The fact that the stiffness matrix are not perfectly symmetric (see Table 4), is due to the small elastic coupling of the NEURARM shoulder and elbow joint, which is caused by the bending of the Bowden cables.

\subsection{Endpoint dynamic behaviour (trajectory following)}

Many neurophysiological studies about motor control of the upper limb have focused their attention on planar motion tasks. As a matter of fact, multi-joint movements require strong interaction between neural, muscular and skeletal system. Consequently, the role of the human arm's 'spring-like' properties becomes a crucial factor for the correct execution of such tasks and has to be taken into account for a proper understanding of the human motor control. In order to perform as a robotic model of the human arm, it is very important that NEURARM be able to replicate planar pointto-point reaching movement by modifying its own intrinsic mechanical properties to verify the resulting effect on the movement dynamics. 
Table 4 Cartesian stiffness matrices estimated by linear regression for five different positions and four different joint stiffness levels combinations Stiffness matrix-experimental characterization

\begin{tabular}{|c|c|c|c|c|c|c|c|c|c|c|c|}
\hline \multirow{4}{*}{$\begin{array}{l}\text { Shoulder }(\mathrm{Nm} / \mathrm{rad}) \\
6.589\left(x_{\mathrm{com}}=1 \mathrm{~mm}\right)\end{array}$} & \multirow{4}{*}{$\begin{array}{l}\text { Elbow }(\mathrm{Nm} / \mathrm{rad}) \\
4.068\left(x_{\mathrm{com}}=1 \mathrm{~mm}\right.\end{array}$} & \multicolumn{10}{|c|}{ End effector position } \\
\hline & & \multicolumn{2}{|c|}{$(0.4,-0.1) \mathrm{m}$} & \multicolumn{2}{|c|}{$(0.4,-0.05) \mathrm{m}$} & \multicolumn{2}{|c|}{$(0.4,0) \mathrm{m}$} & \multicolumn{2}{|c|}{$(0.4,0.05) \mathrm{m}$} & \multicolumn{2}{|c|}{$(0.4,0.1) \mathrm{m}$} \\
\hline & & 167.58 & 15.32 & 153.30 & 6.45 & 151.77 & 51.23 & 131.01 & 58.64 & 109.19 & 64.40 \\
\hline & & 12.86 & 35.88 & 32.45 & 26.74 & 40.03 & 57.15 & 52.01 & 65.29 & 52.04 & 65.29 \\
\hline \multirow[t]{2}{*}{$21.199\left(x_{\mathrm{com}}=5 \mathrm{~mm}\right)$} & $4.068\left(x_{\mathrm{com}}=1 \mathrm{~mm}\right)$ & 255.33 & 60.70 & 219.90 & 31.35 & 198.81 & 98.67 & 159.94 & 116.13 & 136.59 & 112.81 \\
\hline & & 55.96 & 63.79 & 74.98 & 43.14 & 93.82 & 104.14 & 93.96 & 129.63 & 99.07 & 152.74 \\
\hline \multirow[t]{2}{*}{$6.589\left(x_{\mathrm{com}}=1 \mathrm{~mm}\right)$} & $8.422\left(x_{\mathrm{com}}=4 \mathrm{~mm}\right)$ & 249.112 & 5.12 & 232.76 & 1.35 & 227.05 & 51.35 & 195.19 & 72.35 & 192.22 & 83.72 \\
\hline & & 8.35 & 41.38 & 19.28 & 27.79 & 38.68 & 55.02 & 49.84 & 64.10 & 68.59 & 77.67 \\
\hline \multirow[t]{2}{*}{$21.199\left(x_{\mathrm{com}}=5 \mathrm{~mm}\right)$} & $8.422\left(x_{\mathrm{com}}=4 \mathrm{~mm}\right)$ & 281.27 & 26.60 & 260.29 & 32.68 & 266.55 & 87.32 & 237.23 & 110.16 & 203.04 & 117.71 \\
\hline & & 21.90 & 58.59 & 55.07 & 44.26 & 77.85 & 90.13 & 84.62 & 117.98 & 95.91 & 123.69 \\
\hline
\end{tabular}

Fig. 12 Stiffness ellipses estimated perturbing the NEURARM in Cartesian space. The ellipses shape and orientation change (a) for different positions and fixed joint stiffness and (b) for different joint stiffness and fixed position
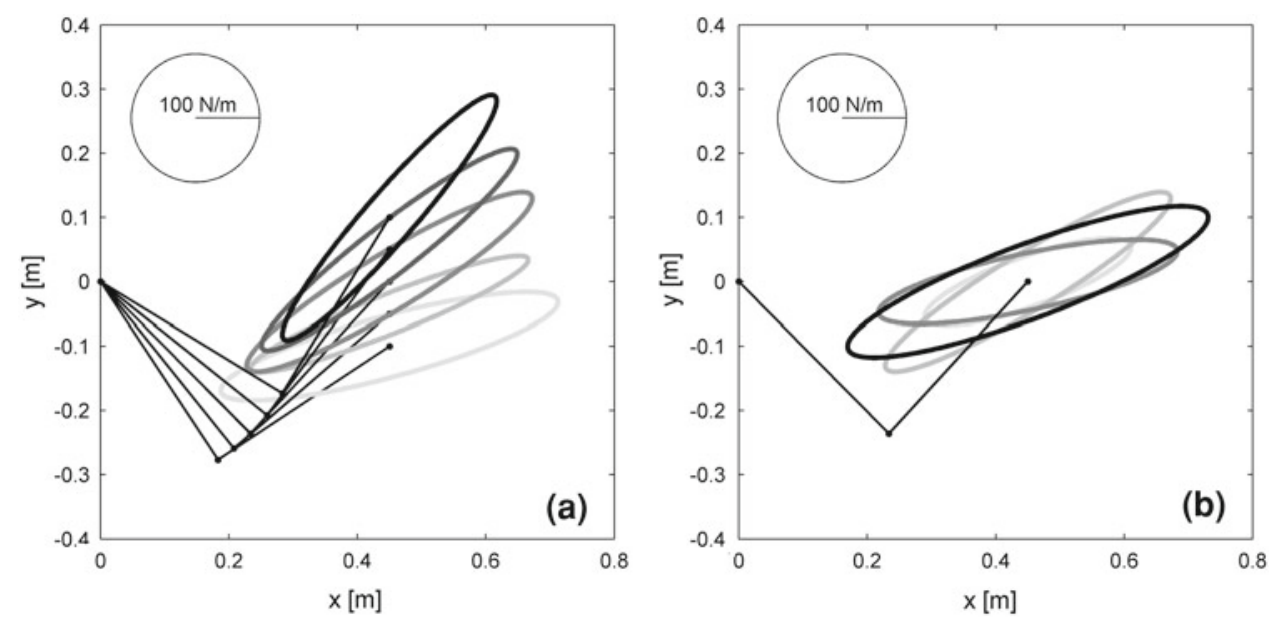

Three different rectilinear paths were tested, as illustrated in Fig. 13, with an equal spatial orientation and distance between the starting and the ending point but different distance from the shoulder joint to provide different joint dynamics. For all the three paths, the $x$ coordinates were moved between -0.2 and $0.2 \mathrm{~m}$ back and forth, whilst the $y$ coordinate was constant for each path and equal respectively to $0.3,0.4$ and $0.5 \mathrm{~m}$. The virtual trajectory (i.e., the desired end effector EP) was calculated to pass from start point to endpoint following a bell-shaped velocity profile, as determined from a minimum jerk trajectory (Flash and Hogan 1985), with a duration of $1 \mathrm{~s}$. Each movement was repeated ten times from start to end and back. The joint stiffness was held constant during the movement. The shoulder stiffness was set to $6.589 \mathrm{Nm} / \mathrm{rad}$ that is $x_{\text {com }}=1 \mathrm{~mm}$, whilst the elbow one was set to $4.068 \mathrm{Nm} / \mathrm{rad}$, which corresponds to $x_{\text {com_ext }}=1 \mathrm{~mm}$; Fig. 13 shows all the performed trajectories, together with the reference paths. The resulting performance is comparable to the numerical simulation performed in Hogan (1984), Flash (1987) and consistent with direct observations on the human being (Gomi and Kawato 1997).

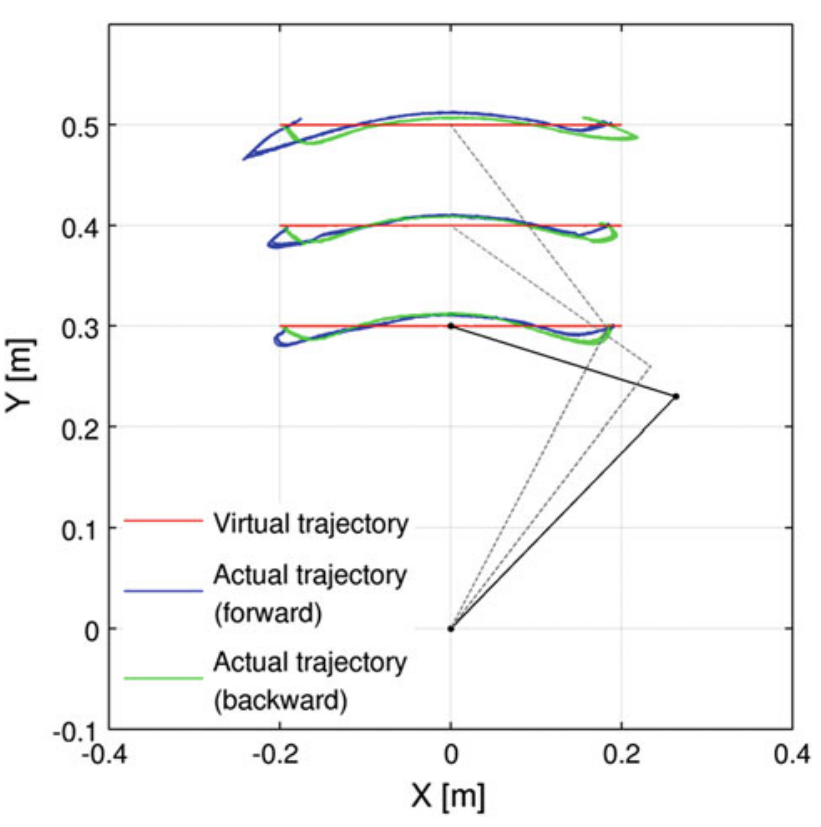

Fig. 13 Cartesian space reaching tasks: three different end point virtual paths are tested by performing ten iteration, back and forth for each trajectory 


\section{Discussion and conclusions}

NEURARM aims to replicate, at least to a certain degree, the apparent mechanical behaviour of the neuromuscular system. Here, we discuss to what extent the NEURARM platform successfully mimics important features of the human musculoskeletal system. We then follow with a discussion of the limitations of the robotic model before drawing some general conclusions about the NEURARM platform as a whole.

\subsection{Characteristics of the human arm successfully mimicked in the NEURARM}

In order to reach this target, we first designed a compliant antagonistic actuation system, which has been used to power each NEURARM joint. A crucial component of the actuation system is the non-linear elastic element, which is designed to mimic the elastic behaviour of muscles acting around a joint, i.e., that stiffness increases with force and torque. We achieved this via a cam mechanism with low friction and inertia which is used to obtain a non-linear force/elongation characteristic, starting from a normal linear tension spring. Measured length-tension (and torque-angle) relationships matched those of the model simulation (see Fig. 7): showing that the system actually achieves the desired stiffness levels.

Two 'muscle-like' actuators in antagonistic configuration provide the basics requirement for developing an open-loop joint position and stiffness control. Both the shoulder and the elbow joint were initially characterized in static conditions. The experimental results (see Fig. 8), show the presence of the convergent torque field about the EP. The slope of the torque field (i.e., the joint stiffness) can be regulated according to the model introduced in Sect. 2.2 (see Table 1). In addition to these static characteristics, the human arm can modulate its stiffness in dynamic conditions, too. NEURARM is able to regulate the stiffness independently from the position: the experimental results (see Fig. 9) confirm that the joint intrinsic mechanical properties can be regulated during dynamic tasks, a result critical for the study of voluntary movements. Moreover, the single joint dynamic performance demonstrates that 'ideally' the arm can move without any feedback by shifting the EP. This is in accordance with biomechanical simulations of the human arm (Flash 1987; Hogan 1984) and with direct observations on deafferented primates (Polit and Bizzi 1978, 1979) and is thought to provide a key element for maintaining stability of movements when interacting with the environment. We verified that the joint stiffness ranges are comparable to that of the human arm whilst performing unconstrained movements in Cartesian space (Gomi and Kawato 1997; Burdet et al. 2000 ). Thus, the first requirement to achieve a suitable robotic model of the human arm is satisfied by our platform.
The analysis of dynamic behaviour at the joints revealed a feature that was not explicitly specified in the design phase. Specifically, we included no requirements about the viscosity around the joints, yet the relationship between stiffness and viscosity has a great effect on the arm performance. In the experimental tests of joint impedance for the NEURARM, we observed that viscosity changed proportionally to the stiffness (see Tables 2,3). This peculiar behaviour is likely due to the Bowden cable transmission and to the antagonistic actuation. Indeed, the more the preloading force is increased (this is needed to regulate the joint stiffness), the higher is the resulting friction (Schiele et al. 2006). As it turns out, this is a fortuitous artifact of the NEURARM design in that the proportional increase of the viscosity along with the stiffness resembles that of the biological system. Indeed, the human arm viscosity is at least proportional to muscles stiffness, as demonstrated by Cannon and Zahalak (1982). Though unexpected, the relationship between viscosity and actuator force of the NEURARM system increases the similarity between the NEURARM and the human limb.

Single-joint tasks do not provide any opportunity to study the effect of the non-linearity introduced by the kinematics of the arm. For this reason most neurophysiological studies focus on tasks involving multiple joints. The NEURARM Cartesian space 'spring-like' properties have been characterized using the same procedure commonly applied to humans (Mussa-Ivaldi and Flash 1990). We showed that, as in humans, the NEURARM elastic behaviour can be described by a stiffness ellipsoid (see Fig. 12). Moreover, the ellipsoids orientation, area and eccentricity changed as a function of the joint position and stiffness (see Table 5), in accordance to what shown by Mussa-Ivaldi et al. (1985) and Gomi and Kawato 1997 on human subjects. As a further evaluation, we also performed dynamic tasks with the NEURARM in Cartesian space. Point-to-point reaching tasks have long been studied because of their importance for understanding the role of the arm elastic properties in motor control. Three different straight trajectories were performed by NEURARM to compare its behaviour with that of the human being. Experimental results (see Fig. 13) showed a good congruence with those obtained by musculoskeletal simulation (Hogan 1984; Flash 1987; Kistemaker et al. 2006) and by direct observations on humans (Gomi and Kawato 1996). Therefore, the NEURARM platform has been shown to be a potential tool for the dynamic analysis of the human arm even during Cartesian space tasks.

\subsection{Scope of the NEURARM model}

Of course, the NEURARM represents a simplified model of the marvellously complex and versatile human arm. Here, we enumerate some of the significant ways in which the NEURARM differs from the human system and discuss how these 
Table 5 Size, orientation and shape of the estimated stiffness ellipses as a function of end effector position and joints stiffness presetting values

Stiffness ellipses-experimental characterization

\begin{tabular}{|c|c|c|c|c|c|c|c|}
\hline \multirow[t]{2}{*}{ Shoulder (Nm/rad) } & \multirow[t]{2}{*}{ Elbow (Nm/rad) } & & \multicolumn{5}{|c|}{ End effector position } \\
\hline & & & $(0.4,-0.1) \mathrm{m}$ & $(0.4,-0.05) \mathrm{m}$ & $(0.4,0) \mathrm{m}$ & $(0.4,0.05) \mathrm{m}$ & $(0.4,0.1) \mathrm{m}$ \\
\hline \multirow{3}{*}{$6.589\left(x_{\mathrm{com}}=1 \mathrm{~mm}\right)$} & \multirow[t]{3}{*}{$4.068\left(x_{\mathrm{com}}=1 \mathrm{~mm}\right)$} & Size $\left(\mathrm{N} / \mathrm{cm}^{2}\right)$ & 1.828 & 1.222 & 2.081 & 1.728 & 1.426 \\
\hline & & Orientation $\left(^{\circ}\right)$ & 5.518 & 14.207 & 19.541 & 28.180 & 34.74 \\
\hline & & Shape & 4.913 & 6.171 & 4.361 & 4.794 & 5.212 \\
\hline \multirow[t]{3}{*}{$21.199\left(x_{\mathrm{com}}=5 \mathrm{~mm}\right)$} & \multirow[t]{3}{*}{$4.068\left(x_{\mathrm{com}}=1 \mathrm{~mm}\right)$} & Size $\left(\mathrm{N} / \mathrm{cm}^{2}\right)$ & 4.050 & 2.242 & 3.596 & 3.085 & 3.042 \\
\hline & & Orientation $\left({ }^{\circ}\right)$ & 15.06 & 21.620 & 31.257 & 37.897 & 40.967 \\
\hline & & Shape & 5.725 & 7.563 & 5.847 & 6.380 & 6.488 \\
\hline \multirow[t]{3}{*}{$6.589\left(x_{\mathrm{com}}=1 \mathrm{~mm}\right)$} & \multirow[t]{3}{*}{$8.422\left(x_{\mathrm{com}}=4 \mathrm{~mm}\right)$} & Size $\left(\mathrm{N} / \mathrm{cm}^{2}\right)$ & 3.135 & 2.024 & 3.300 & 2.797 & 2.886 \\
\hline & & Orientation $\left({ }^{\circ}\right)$ & -2.379 & 5.372 & 11.942 & 17.887 & 24.250 \\
\hline & & Shape & 5.887 & 8.417 & 5.387 & 5.362 & 5.755 \\
\hline \multirow[t]{3}{*}{$21.199\left(x_{\mathrm{com}}=5 \mathrm{~mm}\right)$} & \multirow[t]{3}{*}{$8.422\left(x_{\mathrm{com}}=4 \mathrm{~mm}\right)$} & Size $\left(\mathrm{N} / \mathrm{cm}^{2}\right)$ & 4.994 & 3.054 & 5.411 & 5.864 & 4.343 \\
\hline & & Orientation $\left({ }^{\circ}\right)$ & 5.555 & 13.809 & 20.435 & 26.052 & 32.067 \\
\hline & & Shape & 5.068 & 7.406 & 5.193 & 4.539 & 5.541 \\
\hline
\end{tabular}

differences should be accommodated when comparing the performance of the NEURARM versus humans.

Human muscle is much more complex than what is depicted in Fig. 2. The length-tension characteristics derive from biochemical interactions at the molecular level between the many individual fibres that all work together to produce the macroscopic behaviour of a given muscle. Furthermore, low-level reflex loops act to modulate the lengthtension characteristics, albeit with significant feedback delay. Finally, each joint is actuated by a set of muscles that is typically redundant and the torque produced around the joint is distributed across each of these muscles. As the aim of this study was to produce a robotic model of the human arm that emulates the salient mechanical features of the human neuromuscular system, we did not attempt to replicate the full complexity of the true biological system. Instead, we defined as a design goal the creation of an actuator system that allows for the control of the passive impedance around the joint that works on the joint in an agonist-antagonist configuration and that allows for a range of joint stiffness comparable to what humans can produce, at least for a certain class of voluntary movements.

The two-link planar configuration pales in comparison to the full complexity of the human arm, which has many more degrees of freedom. But it should be noted that considerable insight into human motor behaviour has been gleaned from motion experiments restricted to movements on the horizontal plane (Abend et al. 1982; Gomi and Kawato 1996; Burdet et al. 2001). The NEURARM, therefore, can be used to evaluate any number of current theories about human motor control that are, themselves, limited to the case of two-joint planar arm movements.

Although, the qualitative results are comparable to that of the human arm, it is important to consider that the NEUARM does not have multi-joint muscles. Multi-joint muscles introduce off-diagonal elements in the joint stiffness matrix. Consequently, their absence results in stiffness ellipses being more elongated compared to those of the human arm.

The effect of multi-joint muscles has been subject of interest for a long time (van Ingen Schenau et al. 1987; McIntyre et al. 1996; Gomi and Osu 1996; Osu and Gomi 1999) but still remains open to argument. Thus, it could be an interesting issue to explore on the NEURARM platform. Indeed, it would be possible to simulate the presence of 'virtual' multi joint muscles by a closed-loop control, although the effect of feedback delays in this case should be accurately evaluated. Beyond the 'virtual' implementation, it would be even possible to integrate two further non-linear actuation units on the platform, to emulate the biarticular muscles.

To what extent do the simplifications of the NEURARM with respect to the human arm limit its usefulness in the field of research on human motor control? We would argue that the limitations of the model are manageable, provided that care is taken when interpreting results. If one implements a hypothetical control scheme on the NEURARM and then compares its behaviour to that of humans on a common experimental task, one must of course take special care to assure oneself that the observed difference do not derive from the difference between robot and biology, but this exercise is no more constraining then the mental exercise one must conduct to avoid erroneous conclusions due to erroneous a priori assumptions in any experimental situation. One could further argue that the CNS may also take advantage of simplifying assumptions when generating a control law for the musculoskeletal system. For example, the CNS may ignore as well the detailed force-length-velocity relationships of muscles and instead tune its behaviour based on a simplified internal model of the actuator system's more macroscopic behaviour. Finally, one might argue that certain failures of a simplified model such as the NEURARM to 
reproduce human behaviour can be as revealing as its successes. To give a concrete example, consider the question of multi-joint muscles evoked above. Multi-joint muscles in humans possess the same elastic properties as single-joint muscles, but is this a necessary requirement or a convenient solution? Could Mother Nature has achieved the same results by developing only single-joint muscles coupled by crossjoint reflex loops? This is an example of a situation where one may use the missing feature of the NEURARM robot to prove a point. If stable, human-like behaviour cannot be achieved without the existence of elastic multi-joint actuators, this fact would help us to understand the reason that multi-joint muscles evolved in the first place. This is one example of the type of research we plan to pursue using the NEURARM platform.

\subsection{Conclusions}

The aim of this study was to achieve a robotic model of the human arm able to replicate the apparent mechanical behaviour of the neuromuscular system to investigate their role in motor control. To this end, an antagonistic 'muscle-like' actuation system has been designed and developed. A nonlinear elastic element, replicating the mechanical elasticity of the muscle-tendon complex, has been designed accordingly to a mathematical model to optimize the achievable stiffness range. The final prototype has been characterized to confirm that the design goals have been met. The 'muscle-like' actuation system has been integrated on the NEURARM: an extensive static and dynamic characterization of the robot has been performed in both joint and Cartesian space. The experimental results pointed out that the NEURARM performance is sufficiently close to those of the human arm for certain key features, both in static and dynamic conditions. Therefore, the NEURARM proves to be a suitable robotic model of the human arm and, as such, it could represent a possible innovative and powerful tool for neuroscience investigation.

Acknowledgments Authors would like to thank Dr. Fabrizio Vecchi and Mr. Francesco Giovacchini for their precious suggestions during all the experimental activities. This study was partly supported by the EU within the NEUROBOTICS Integrated Project (The fusion of NEUROscience and roBOTICS, IST-FET Project \#2003-001917) and within the EVRYON Collaborative Project STREP (Evolving Morphologies for Human-Robot Symbiotic Interaction, Project FP7-ICT-20073-231451)

Open Access This article is distributed under the terms of the Creative Commons Attribution Noncommercial License which permits any noncommercial use, distribution, and reproduction in any medium, provided the original author(s) and source are credited.

\section{References}

Abend W, Bizzi E, Morasso P (1982) Human arm trajectory formation. Brain 105:331-348
Bizzi E, Accornero N, Chapple W, Hogan N (1984) Posture control and trajectory formation during arm movement. J Neurosci 4:27382744

Burdet E, Osu R, Franklin DW, Milner TE, Yoshioka T, Milner T, Kawato M (2000) A method for measuring end-point stiffness during multi-joint arm movement. J Biomech 33(12):1705-1709

Burdet E, Osu R, Franklin DW, Milner TE, Kawato M (2001) The central nervous system stabilizes unstable dynamics by learning optimal impedance. Nature 414:446-449

Caldwell DG, Medrano-Creda GA, Goodwin M (1995) Control of pneumatic muscle actuators. IEEE Control Syst Mag 15(1):40-48

Cannon S, Zahalak G (1982) The mechanical behavior of active human skeletal muscle in small oscillation. J Biomech 15:111-121

Cattin E, Roccella S, Vitiello N, Clemens E, Sardellitti I, Panagiotis KA, Vacalebri P, Vecchi F, Carrozza MC, Kyriakopulos K, Dario P (2008) Design and development of a novel robotic platform for neuro-robotics applications: the NEURobotics ARM (NEURARM). Adv Robot. Special issue on robotics platforms for neuroscience, 22:3-37

Colgate JE, Hogan N (1988) Robust control of dynamically interacting systems. Int J Control 48:65-88

Feldman AG (1966) Functional tuning of the nervous system with control of movement or maintenance of steady posture, II: controllable parameters of the muscles. Biophysics 11:565-578

Feldman AG (1986) Once more on the equilibrium-point hypothesis ( $\lambda$ model) for motor control. J Mot Behav 18(1):17-54

Filippini R, Sen S, Bicchi A (2008) Toward soft robot you can depend on. IEEE Robot Autom Mag. 15(3):31-41

Flash T (1987) The control of hand equilibrium trajectories in multijoint arm movement. Biol Cybern 57:257-274

Flash T, Hogan N(1985) The coordination of arm movements: an experimentally confirmed mathematical model. J Neurosci 5(7):1688-1703

Gomi H, Kawato M (1996) Equilibrium-point control hypothesis examined by measured arm stiffness during multijoint movement. Science 272:117-120

Gomi H, Kawato M (1997) Human arm stiffness and equilibrium-point trajectory during multi-joint movement. Biol Cybern 76:163-171

Gomi H, Osu R (1996) Contributions of single and double joint stiffness of human arm during force control. In: Proceedings of the 18th annual international conference of the IEEE, vol 5. Engineering in Medicine and Biology Society, Amsterdam, pp 2244-2245

Hogan N (1984) An organizing principle for a class of voluntary movements. J Neurosci 4:2745-2754

Hogan N (1985a) Impedance control: an approach to manipulation. Part I: theory. ASME J Dyn Syst Control 107:1-7

Hogan N (1985b) Impedance control: an approach to manipulation. Part II: implementation. ASME J Dyn Syst Control 107:8-16

Hogan N (1985c) Impedance control: an approach to manipulation. Part III: application. ASME J Dyn Syst Control 107:17-24

Hogan N, Bizzi E, Mussa-Ivaldi FA, Flash T (1987) Controlling multijoint motor behaviour. Exerc Sport Sci Rev 15:153-190

Kistemaker DA, Van Soest AKJ, Bobbert F (2006) Is equilibrium point feasible for fast goal-directed single-joint movement?. J Neurophysiol 95:2898-2912

Koganezawa K, Ianba T, Nakazawa T (2006) Stiffness and angle control of antagonistically driven joint. In: Proceedings of the IEEE international conference on biomedical robotics and biomechatronics, pp 1007-1013

Lenzi T, De Rossi S, Vitiello N, Chiri A, Roccella S, Giovacchini F, Vecchi F, Carrozza MC (2009) The neuro-robotics paradigm: NEURARM, NEUROExos, HANDEXOS, In: Proceedings of the annual international conference of the IEEE engineering in medicine and biology society EMBC 2009, 3-6 Sep, pp 2430-2433

McIntyre J, Mussa-Ivaldi FA, Bizzi E (1996) The control of stable postures in the multijoint arm. Exp Brain Res 110:248-264 
Migliore SA, Brown EA, DeWeerth SP (2005) Biologically inspired joint stiffness control. In: Proceedings of the IEEE international conference on robotics and automation, pp 2171-2176

Mussa-Ivaldi F, Flash T (1990) Human arm stiffness characteristics during the maintenance of posture. Exp Brain Res 82(2):315-326

Mussa-Ivaldi FA, Hogan N, Bizzi E (1985) Neural, mechanical, and geometric factors subserving arm posture in humans. J Neurosci 5:2732-2743

Osu R, Gomi H (1999) Multijoint muscle regulation mechanisms examined by measured human arm stiffness and EMG signals. J Neurophysiol 81:1458

Polit A, Bizzi E (1978) Processes controlling arm movements in monkeys. Science 201:1235-1237

Polit A, Bizzi E (1979) Characteristics of motor programs underlying arm movements in monkeys. J Neurophysiol 42:183-194

Schiele A, Letier P, van der Linde R, van der Helm F (2006) Bowden cable actuator for force-feedback exoskeletons. In: Proceedings of the 2006 IEEE international conference on intelligent robots and systems, Beijing, 9-15 Oct, pp 3599-3604

Selden B, Cho K-J, Asada H (2006) Segmented shape memory alloy actuators using hysteresis loop control. J Smart Mater Struct 15(2):642-652
Tondu B, Ippolito S, Guiochet J, Daidie A (2005) A seven-degreeof-freedom robot-arm driven by pneumatic artificial muscle for humanoid robots. Int J Robot Res 24(4):257-274

van Ingen Schenau G, Bobbert M, Rozendal R (1987) The unique action of bi-articular muscles in complex movements. J Anat 155:1

Vitiello N, Cattin E, Roccella S, Giovacchini F, Vecchi F, Carrozza MC, Dario P (2007) The NEURARM towards a platform for joint neuroscience on human motion control theories. In: Proceedings of the 2007 IEEE international conference on intelligent robots and systems, San Diego, 29 Oct-2 Nov, pp 1852-1857

Vitiello N, Lenzi T, McIntyre J, Roccella S, Cattin E, Vecchi F, Carrozza MC (2008) Characterization of the NEURARM bio-inspired joint position and stiffness open loop controller. In: Proceedings of the 2008 IEEE international conference on biomedical robotics and biomechatronics, Scottsdale, Az, 19-22 Oct, pp 138-143

Vitiello N, Lenzi T, De Rossi SMM, Roccella S, Carrozza MC (2010) A sensorless torque control for antagonistic driven compliant joints. Mechatronics 20(3):355-367 